\title{
Newhouse regions for reversible systems with infinitely many stable, unstable, and elliptic periodic orbits
}

\author{
Jeroen S.W. Lamb and Oleg V. Stenkin * \\ Department of Mathematics \\ Imperial College \\ London SW7 2BZ, UK
}

February 24, 2004

\section{Contents}

1 Introduction and statement of the main results. 1

2 Geometric construction $\quad 6$

2.1 Local and global maps . . . . . . . . . . . . . . . . . . . . . . . . 6

2.2 Geometric Tools . . . . . . . . . . . . . . . . . . . . . . 9

2.3 Dynamics near heteroclinic cycles . . . . . . . . . . . . . . . . . . 13

3 Newhouse regions for homoclinic and heteroclinic tangencies 14

3.1 Density of diffeomorphisms with homoclinic tangencies . . . . . . . . . . . . . 15

3.2 Density of diffeomorphisms with symmetric heteroclinic cycles . . . . . . . . . . . 16

3.3 Density of diffeomorphisms with non-symmetric heteroclinic cycles . . . . . . . . . . 18

4 Bifurcations of single-round periodic orbits $\quad 19$

4.1 Construction of the Poincaré map $T_{i i}$ and Rescaling Lemma. . . . . . . . . . . . 19

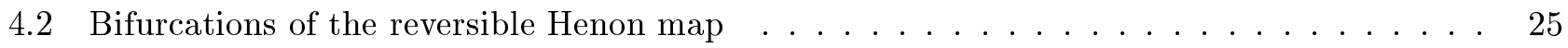

4.3 Bifurcations of non-symmetric periodic orbits. Rescaling lemma for the map $T_{i j} \ldots \ldots$. . 25

4.4 Diffeomorphisms with infinitely many stable, unstable and elliptic periodic orbits in New-

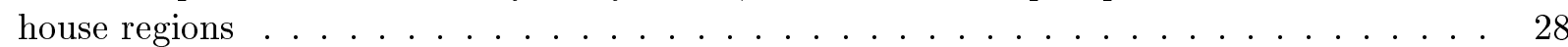

\begin{abstract}
For reversible two-dimensional diffeomorphisms we establish a new type of Newhouse regions (regions of structurally instability density). We prove that in these regions there exists a dense set of diffeomorphisms having simultaneously infinitely many stable, infinitely many unstable and infinitely many elliptic type periodic orbits.
\end{abstract}

\section{Introduction and statement of the main results.}

One of the most important results of bifurcation theory of dynamical systems is the discovery of regions of structural instability in the space of smooth systems. Examples of these regions are the regions in which there exists a dense set of systems with saddle periodic orbits having tangencies of their stable and

\footnotetext{
${ }^{*}$ permanent address: Research Institute for Applied Mathematics and Cybernetics, Nizhny Novgorod, Russia
} 
unstable manifolds along homoclinic orbits. This fact was established by Newhouse [27, 26] in the case of two-dimensional diffeomorphisms. Later, this result was extended to higher dimensions in [17, 28, 30]. Naturally, such regions, both in the space of dynamical systems and in the parameter space, have been called Newhouse regions.

Dynamical properties of systems in Newhouse regions are very complicated. In order to describe the bifurcation phenomena of such systems, infinitely many parameters are required. This is connected with the fact that in Newhouse regions diffeomorphisms with periodic (and homoclinic) orbits of any order of degeneracy are dense $[19,18,13,16]$. Another important property of systems in Newhouse regions is the presence of infinitely many stable (or unstable) periodic orbits $[5,25,18]$. Note, that such type Newhouse regions appear in generic one-parameter families which are transverse to a codimension one bifurcation surface, consisting of diffeomorphisms with homoclinic tangencies of manifolds of dissipative saddle fixed points.

An essentially other type of Newhouse regions arises near diffeomorphisms with a non-transversal heteroclinic cycle consisting of several saddle fixed (periodic) points and heteroclinic orbits. In the (codimension one) case when the heteroclinic cycle contains only one non-transversal heteroclinic orbit with a quadratic tangency of corresponding stable and unstable manifolds and the saddle values of one of fixed points is less than one, and the saddle value of another is greater than one, a new phenomenon was established [20]: there exist Newhouse regions in which diffeomorphisms that have simultaneously infinitely many saddle, stable and completely unstable periodic orbits are dense. This assertion also holds for generic one parameter families. Moreover, in the context of two parameter analysis allows to prove the density of diffeomorphisms with infinitely many stable and unstable invariant closed curves [12]. In these situations, it is naturally to speak of Newhouse regions (intervals) with mixed dynamics. Note that in these regions diffeomorphisms with homoclinic tangencies are also dense and, consequently, diffeomorphisms with above mentioned structurally unstable periodic and homoclinic orbits are dense.

The aim of the present paper is to describe the properties of Newhouse regions which can exist in two-dimensional reversible diffeomorphisms. Recall that a diffeomorphism $f: \mathbb{R}^{2} \mapsto \mathbb{R}^{2}$ is called reversible if there exists a diffeomorphism $R: \mathbb{R}^{2} \mapsto \mathbb{R}^{2}$, which conjugates $f$ to its inverse $f^{-1}$, ie

$$
R \circ f \circ R^{-1}=f^{-1},
$$

see for instance [22].

In this paper, we will confine ourselves to the case that $R^{2}=I, f$ is orientation preserving $(\operatorname{det} d f>0)$, and $R$ is orientation reversing ( $\operatorname{det} d R<0)$. Let $\operatorname{Fix}(\mathrm{R})$ denote the set of fixed points of $R$. It follows from our assumptions that $\operatorname{Fix}(\mathrm{R})$ and $\operatorname{Fix}(\mathrm{f} \circ \mathrm{R})$ have dimension 1. For such reversible two-dimensional diffeomorphisms it is well known that symmetric ( $R$-invariant) periodic orbits are generically either of saddle type (one attracting and one repelling multiplier) or elliptic (two multipliers on the complex unit circle). Asymmetric periodic orbits arise pairwise: they either form $R$-invariant pairs of stable and completely unstable periodic orbits, or pairs of saddle periodic orbits (where typically the Jacobian is greater than 1 for one of them and less than 1 for the other one).

We consider generic one parameter families of two-dimensional reversible diffeomorphisms containing a diffeomorphism with a non-transversal heteroclinic cycle consisting of two saddle fixed points and two heteroclinic orbits, one of which is non-transversal. We show that in any parameter neighborhood of the parameter value corresponding to the non-transversal heteroclinic cycle, there are Newhouse parameter intervals where diffeomorphisms having simultaneously infinitely many stable, completely unstable, saddle and elliptic type periodic points, are dense.

We now make our assumptions more precise. Let $f_{\mu}$ be a one $(\mu)$ parameter family of planar $C^{r}$-smooth $(r \geq 5) R$-reversible diffeomorphisms, where $R$ is orientation reversing and $f$ orientation preserving. We suppose that

[H1] $f_{\mu}$ has a saddle fixed point $O_{1} \notin \mathrm{Fix}(\mathrm{R})$, with multipliers $\lambda, \gamma,|\lambda|<1<|\gamma|$. It follows from reversibility that $f$ has another saddle fixed point $O_{2}=R\left(O_{1}\right)$ with multipliers $\gamma^{-1}, \lambda^{-1}$, $\left|\gamma^{-1}\right|<1<\left|\lambda^{-1}\right|$. 
[H2] We suppose that the Jacobians $\sigma_{i}=\operatorname{det}\left(d f_{0}\left(O_{i}\right)\right)$, with $i=1,2$, are not equal to 1 . Note that $\sigma_{1}=\lambda \gamma ; \sigma_{2}=(\lambda \gamma)^{-1}$.

[H3] The diffeomorphism $f_{0}$ has two heteroclinic orbits, $\Gamma_{12}$ and $\Gamma_{21}$, such that $\Gamma_{12} \subset W^{u}\left(O_{1}\right) \cap W^{s}\left(O_{2}\right)$ and $\Gamma_{21} \subset W^{u}\left(O_{2}\right) \cap W^{s}\left(O_{1}\right)$. We suppose that

1) $W^{u}\left(O_{1}\right)$ and $W^{s}\left(O_{2}\right)$ intersect transversally at the points of orbit $\Gamma_{12}$

2) $W^{u}\left(O_{2}\right)$ and $W^{s}\left(O_{1}\right)$ have a quadratic tangency at the points of orbit $\Gamma_{21}$,

3) $R\left(\Gamma_{12}\right)=\Gamma_{12}, R\left(\Gamma_{21}\right)=\Gamma_{21}$.

The set $C=\left\{O_{1}, O_{2}, \Gamma_{12}, \Gamma_{21}\right\}$ is a heteroclinic cycle. We call such a cycle a non-transversal symmetric heteroclinic cycle, as it is non-transversal by 2) and $R$-invariant by 3 ). There turn out to be four different types of such cycles. For the case of positive multipliers they are sketched in Fig. 1.

The diffeomorphisms which are close to $f_{0}$ and satisfy the conditions [H1-H3] form a locally connected surface $H$ of codimension 1 in the space of reversible two-dimensional diffeomorphisms. In this paper we study one-parameter families $f_{\mu}$, where $\mu$ is a parameter governing the splitting of the manifolds $W^{u}\left(O_{2}\left(\mu_{1}\right)\right)$ and $W^{s}\left(O_{1}\left(\mu_{1}\right)\right)$. We thus assume that

[H4] $f_{\mu}$ is transversal to $H$.

Our main result is summarized in the following theorem:

Theorem 1 Let $f_{\mu}$ be a one parameter family of R-reversible planar diffeomorphisms as discussed above, satisfying [H1-H4]. Then, for any $\mu_{0}>0$, the interval $I=\left(-\mu_{0}, \mu_{0}\right)$ contains a countable set of intervals $\delta_{j} \subset I$ of values of $\mu$ such that $\delta_{j} \rightarrow 0$ as $j \rightarrow \infty$ and

1) in $\delta_{j}$ there is dense set of values of $\mu, \mu=\mu^{*}$, at which the diffeomorphism $f_{\mu}$ has a non-transversal homoclinic orbit $\Gamma_{1}(\mu)$ to $O_{1}$ (and consequently also a non-transversal homoclinic orbit $\Gamma_{2}(\mu)=$ $R \Gamma_{1}(\mu)$ to $\left.O_{2}=R\left(O_{1}\right)\right)$. Moreover, the parameter $\mu-\mu^{*}$ splits this tangency monotonically.

2) in $\delta_{j}$ there is a dense set of values of $\mu, \mu=\mu^{*}$, at which the diffeomorphism $f_{\mu^{*}}$ has a symmetric type non-transversal heteroclinic cycle consisting of fixed points $O_{1}\left(\mu^{*}\right), O_{2}\left(\mu^{*}\right)$, a transversal heteroclinic orbit $\Gamma_{12}\left(\mu^{*}\right)$ and a non-transversal heteroclinic orbit $\Gamma_{21}^{j}\left(\mu^{*}\right)$ in whose points the manifolds $W^{u}\left(O_{2}\left(\mu^{*}\right)\right)$ and $W^{s}\left(O_{1}\left(\mu^{*}\right)\right)$ have a quadratic tangency; the parameter $\mu-\mu^{*}$ splits this tangency monotonically.

3) in $\delta_{j}$ there is a dense set of values of $\mu, \mu=\mu^{*}$, at which diffeomorphism $f_{\mu^{*}}$ has a non-symmetric type non-transversal heteroclinic cycle consisting of orbits $O_{1}\left(\mu^{*}\right), O_{2}\left(\mu^{*}\right), \Gamma_{12}\left(\mu^{*}\right)$ and $\Gamma_{21}^{j}\left(\mu^{*}\right) ;$ the parameter $\mu-\mu^{*}$ splits this tangency monotonically.

4) in $\delta_{j}$ there is a dense set of values of $\mu$, at which diffeomorphism $f_{\mu}$ has infinitely many saddle, infinitely many stable, infinitely many completely unstable periodic orbits and infinitely many elliptic type periodic points, whose closure contains the fixed points $\mathrm{O}_{1}$ and $\mathrm{O}_{2}$.

In analogy to Newhouse's result on the persistence of homoclinic tangencies [26, 17], for the one parameter families under consideration we find a dense set of diffeomorphisms with homoclinic tangencies in parameter intervals $\delta_{j}$. Accordingly, we call $\delta_{j}$ Newhouse intervals. However, we prove that in our context of reversible systems, the dynamics of the diffeomorphisms is mixed, with elliptic, stable, and unstable behaviour.

Note that in area-preserving case dynamical properties of diffeomorphisms in Newhouse regions have been studied by Duarte [4]. In particular, in [3], the existence of infinitely many elliptic orbits were found for area-preserving Henon maps. Gonchenko, Shilnikov and Turaev [14, 15] discuss generic 2-elliptic 

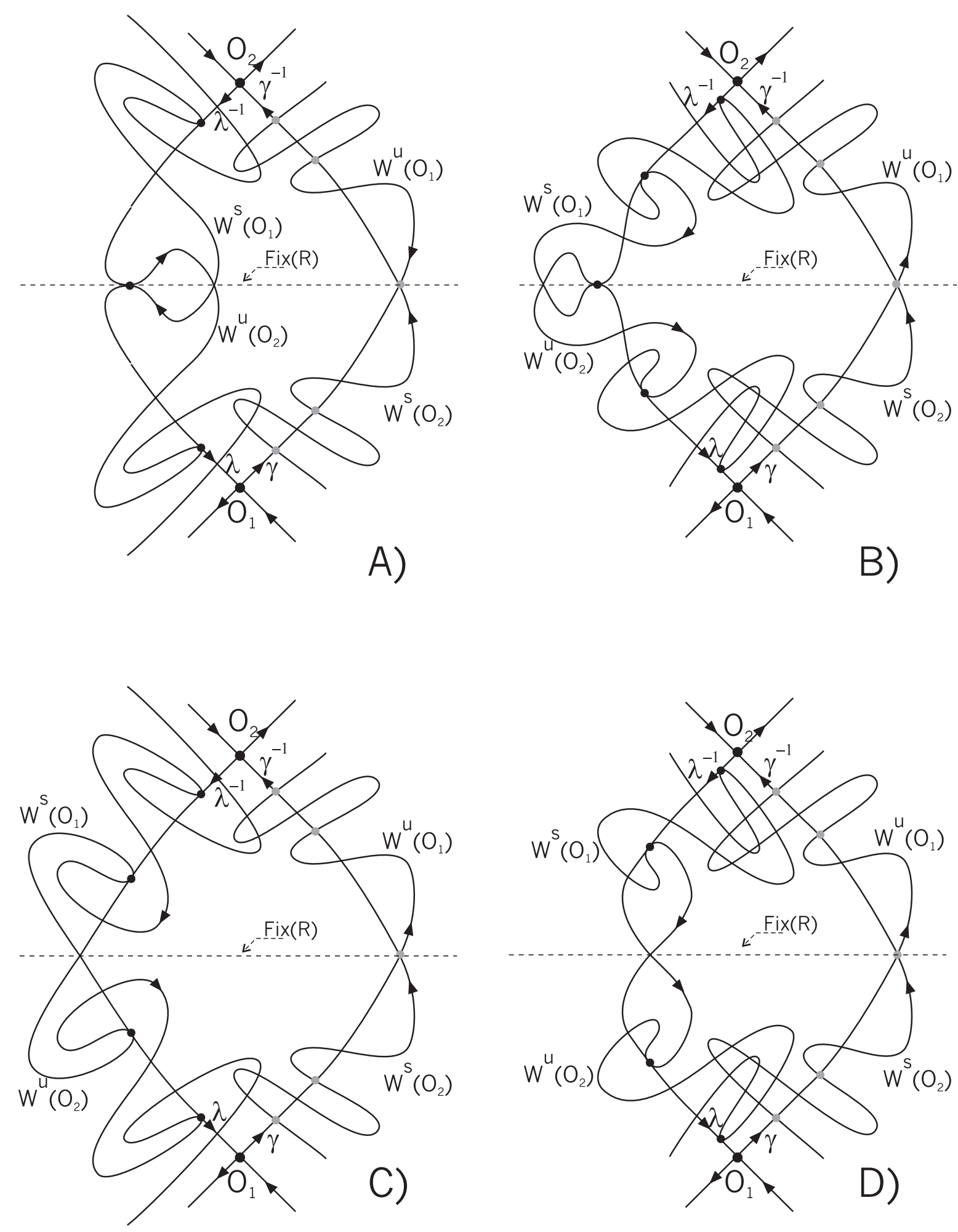

Figure 1: Various types of diffeomorphism with non-transversal heteroclinic cycle 
periodic orbits at four-dimensional symplectic maps close to a map with a non-transversal homoclinic orbit to a saddle-focus fixed point. The phenomenon of "mixed dynamics" for area-preserving twodimensional diffeomorphisms was also discussed in [10, 11].

To our knowledge, our paper is the first to discuss Newhouse regions for reversible diffeomorphisms, revealing the novel phenomenon of the simultaneous occurrence of infinitely many stable, completely unstable and elliptic periodic orbits. Importantly, although it is well known that elliptic, saddle, attracting and repelling periodic points can coexist in reversible diffeomorphisms, it is far from obvious that infinities of those should coexist persistently in the neighbourhood of symmetric heteroclinic tangencies. Moreover, by the simultaneous creation of symmetric (saddle, elliptic) and nonsymmetric (attractors, repellers) periodic orbits, the heteroclinic bifurcation thus induces some degree of symmetry breaking, by having close to the non-transversal cycle both symmetric and nonsymmetric orbits, as well as nonsymmetric tangencies.

In the literature, homoclinic and heteroclinic orbits in reversible systems have most frequently been studied in the context of ODEs, where the connecting orbits arise between equilibria, see for instance $[2,21,23]$. Our study relates to the study of dynamics near heteroclinic orbits in reversible systems between a pair of asymmetric periodic solutions of saddle type. In fact, an important class of reversible ODEs are those derived from a $\mathbb{Z}_{2}$-symmetric PDE $[2,21,23]$, by restriction to travelling and/or standing waves. In this context, our results can be interpreted to shed light on the complicated structure of the solution set of the PDE in the neighbourhood of heteroclinic cycles between periodic waves.

Two-dimensional reversible diffeomorphisms appear naturally as Poincaré maps for symmetric periodic orbits in three-dimensional reversible vector fields. Such a situation arises for instance in the travelling/standing wave reduction of the Kuramoto-Shivashinsky PDE [24, 23]. Alternatively, such reversible maps can appear naturally when periodically forced reversible flows are considered where the forcing has a special form (eg odd or even function of time).

We now proceed with discussing yet another example from the recent physics literature where reversible two-dimensional maps having non-transversal heteroclinic cycles appear as Poincare maps. Topaj and Pikovsky [29] describe the dynamics of four coupled rotators, by the following set of differential equations

$$
\begin{aligned}
& \dot{\psi}_{1}=1-2 \varepsilon \sin \psi_{1}+\varepsilon \sin \psi_{2} \\
& \dot{\psi}_{2}=1-2 \varepsilon \sin \psi_{2}+\varepsilon \sin \psi_{1}+\varepsilon \sin \psi_{3} \\
& \dot{\psi}_{3}=1-2 \varepsilon \sin \psi_{3}+\varepsilon \sin \psi_{2}
\end{aligned}
$$

where the $\psi_{i}$ denote phase differences and $\varepsilon$ is the coupling coefficient. This system is reversible with respect to the involution $\left(\psi_{1}, \psi_{2}, \psi_{3}\right) \rightarrow\left(\pi-\psi_{3}, \pi-\psi_{2}, \pi-\psi_{1}\right)$. When $\varepsilon$ is small, the three-dimensional flow (1) can be reduced to the following non-autonomous periodically forced two-dimensional flow

$$
\begin{aligned}
\dot{\xi} & =\frac{2 \varepsilon \sin \xi \sin \eta}{2+\varepsilon \cos (t-\eta)} \\
\dot{\eta} & =\frac{1-\varepsilon \cos (t-\eta)-2 \varepsilon \cos \xi \cos \eta}{2+\varepsilon \cos (t-\eta)}
\end{aligned}
$$

which is reversible with respect to the involution $(\xi, \eta) \rightarrow(\xi,-\eta)$. Numerical experiments from [29, 6] indicates that the Poincaré map can display a heteroclinic cycle with visable traces of the Newhouse phenomenon, as is studied in detail in the present paper. Illustrations in [29] may be interpreted as the existence of "intersection" of attractors and repellers, i.e. the closure of sets of stable and completely unstable periodic orbits containig hyperbolic subsets. For more on this, see $[29,6])$.

This paper is organized as follows. In Section 2 we discuss the geometric construction underlying our proof of Theorem 1. We discuss also the classification of diffeomorphisms with a non-transversal heteroclinic cycle and point out the principal difference with the non-reversible case. To study the main bifurcations of such diffeomorphisms we consider general one-parameter unfoldings. In Section 3 we prove the statements $1-3$ of Theorem 1. In Section 4 we prove the first three statements of Theorem 1. 


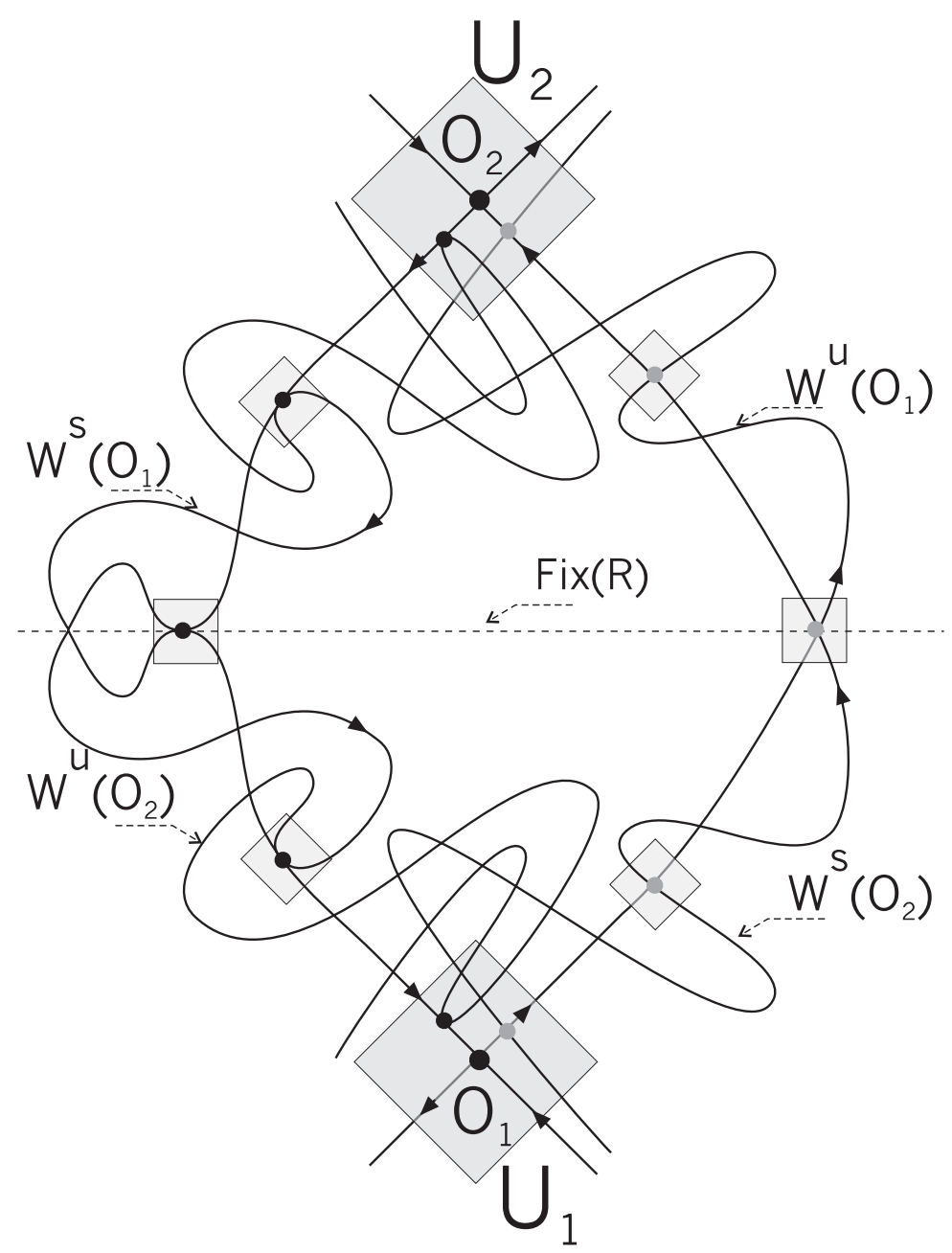

Figure 2: The neighbourhood of a non-transversal symmetric heteroclinic cycle

In order to prove density of diffeomorphisms with mixed types of periodic orbits we consider bifurcations of single-round periodic orbits. In particular, in subsection 4.1 (the Rescaling Lemma) we reduce the study of the corresponding Poincaré map to the study of the reversible Henon map. It allows us to find diffeomorphisms with elliptic periodic points in the subsection 4.2. Subsection 4.3 is devoted to prove the existence of diffeomorphisms with stable and unstable periodic orbits. We reduce this problem to the study of bifurcations of a parabola map. Finally, in subsection 4.4 we summarize our results and prove the fourth and final statement of Theorem 1.

\section{Geometric construction}

We a consider $C^{r}$-smooth $(r \geq 5)$, two-dimensional reversible diffeomorphism $f_{0}$ having a symmetric nontransversal heteroclinic cycle $C$ as detailed in Section 1. Consider a sufficiently small fixed neighbourhood $U$ of the cycle $C$. $U$ is a union of two discs $U_{1}$ and $U_{2}$ containing the points $O_{1}$ and $O_{2}$, and a number of discs containing points of orbits $\Gamma_{12}$ and $\Gamma_{21}$ which lie outside $U_{1}$ and $U_{2}$, see Fig. 2 for a sketch.

\section{$2.1 \quad$ Local and global maps}

Denote by $T_{0 l}(\mu), l=1,2$, the restriction of $f_{\mu}$ onto $U_{l}$, i.e. $\left.T_{0 l}(\mu) \equiv f_{\mu}\right|_{U_{l}}$. We will call maps $T_{0 l}(\mu)$ and $T_{02}(\mu)$ the local maps. Consider the neighbourhood $U_{1}$. It is known $[8,9,7]$ that there exist $C^{r-1}$ - 
coordinates on $U_{1}$ such that $T_{0 l}(\mu)$ can be written in the form

$$
\begin{aligned}
& \bar{x}_{1}=\lambda(\mu) x_{1}+g_{1}\left(x_{1}, y_{1}, \mu\right) x_{1}^{2} y_{1}, \\
& \bar{y}_{1}=\gamma(\mu) y_{1}+g_{2}\left(x_{1}, y_{1}, \mu\right) x_{1} y_{1}^{2},
\end{aligned}
$$

where the right sides of (2) are $C^{r-1}$ (in coordinates and parameters). In these coordinates, for sufficiently small $\mu$, the point $O_{1}(\mu)$ has coordinates $x_{1}=0, y_{1}=0$ and the equations of $W_{l o c}^{s}\left(O_{1}(\mu)\right)$ and $W_{l o c}^{u}\left(O_{1}(\mu)\right)$ are $y_{1}=0$ and $x_{1}=0$, respectively. Of course, the coordinates $\left(x_{1}, y_{1}\right)$ are only defined in the small region $U_{1}$ of $O_{1}$. Let $(u, v)$ denote the natural global coordinates in $\mathbb{R}^{2}$. Then there is a coordinate transformation $q: U_{1} \rightarrow \mathbb{R}^{2}$

$$
\left(\begin{array}{l}
u \\
v
\end{array}\right)=q\left(\begin{array}{l}
x_{1} \\
y_{1}
\end{array}\right)
$$

The representation (2) of $T_{01}$ in the local coordinates $\left(x_{1}, y_{1}\right)$ is given by

$$
T_{01}=q^{-1} \circ f \circ q
$$

Now we choose coordinates $\left(x_{2}, y_{2}\right)$ in $U_{2}$ such that

$$
\left(\begin{array}{l}
u \\
v
\end{array}\right)=\tilde{q}\left(\begin{array}{c}
x_{2} \\
y_{2}
\end{array}\right)
$$

where $\tilde{q}: U_{2} \rightarrow \mathbb{R}^{2}, \tilde{q}=R \circ q \circ R_{12}^{-1}$, and $R_{12}: U_{1} \rightarrow U_{2}$ is some smooth diffeomorphism. By reversibility of $f$ we have $R^{-1} \circ f^{-1} \circ R=f$, so that

$$
T_{01}=q^{-1} \circ f \circ q=q^{-1} \circ R^{-1} \circ f^{-1} \circ R \circ q=R_{12}^{-1} \circ \tilde{q}^{-1} f^{-1} \circ \tilde{q} \circ R_{12} .
$$

Analogously, in local coordinates we have

$$
T_{02}=\tilde{q}^{-1} \circ f \circ \tilde{q},
$$

and hence

$$
T_{01}=R_{12}^{-1} \circ T_{02}^{-1} \circ R_{12} .
$$

It is convenient to choose $R_{12}$ as $R_{12}\left(x_{1}, y_{1}\right)=\left(y_{2}, x_{2}\right)$. By virtue of $(2)$ and (3), the local map $T_{02}^{-1}(\mu)$ will thus have the form

$$
\begin{aligned}
& y_{2}=\lambda(\mu) \bar{y}_{2}+f\left(\bar{y}_{2}, \bar{x}_{2}, \mu\right) \bar{y}_{2}^{2} \bar{x}_{2}, \\
& x_{2}=\gamma(\mu) \bar{x}_{2}+g\left(\bar{y}_{2}, \bar{x}_{2}, \mu\right) \bar{y}_{2} \bar{x}_{2}^{2},
\end{aligned}
$$

Consider again the diffeomorphism $f_{0}$. The point $O_{1}$ is an $\alpha$-limit point for $\Gamma_{12}$ and an $\omega$-limit point for $\Gamma_{21}$; the point $O_{2}$ is an $\alpha$-limit point for $\Gamma_{21}$ and an $\omega$-limit point for $\Gamma_{12}$. Hence, countable sets of heteroclinic points (points of orbits $\Gamma_{12}$ and $\left.\Gamma_{21}\right)$ lie on $W_{l o c}^{s}\left(O_{l}\right)$ and $W_{l o c}^{u}\left(O_{l}\right)$. Since $\Gamma_{12}$ and $\Gamma_{21}$ are symmetric, i.e. $R\left(\Gamma_{12}\right)=\Gamma_{12}$ and $R\left(\Gamma_{21}\right)=\Gamma_{21}$ we can choose two pairs of the symmetric heteroclinic points: $M_{2}^{+}\left(x_{2}^{+}, 0\right) \in U_{2}$ and $M_{1}^{-}\left(0, y_{1}^{-}\right) \in U_{1}$ of the orbit $\Gamma_{12}$ and $M_{1}^{+}\left(x_{1}^{+}, 0\right) \in U_{1}$ and $M_{2}^{-}\left(0, y_{2}^{-}\right) \in U_{2}$ of the orbit $\Gamma_{21}$, where $x_{1}^{+}=y_{2}^{-}$and $x_{2}^{+}=y_{1}^{-}$. Without loss of generality, we may choose $x_{1}^{+}>0$. Let $n_{1}$ and $n_{2}$ be positive integers such that $f_{0}^{n_{1}}\left(M_{1}^{-}\right)=M_{2}^{+}$and $f_{0}^{n_{2}}\left(M_{2}^{-}\right)=M_{1}^{+}$. Consider sufficiently small neighbourhoods $\Pi_{l}^{+} \subset U_{l}$ and $\Pi_{l}^{-} \subset U_{l}$ of points $M_{l}^{+}$and $M_{l}^{-}$, respectively. Then, the global maps $T_{12}(\mu) \equiv f_{\mu}^{n_{1}}: \Pi_{1}^{-} \rightarrow U_{2}$ and $T_{21}(\mu) \equiv f_{\mu}^{n_{2}}: \Pi_{2}^{-} \rightarrow U_{1}$ are defined for all $\mu$ small.

Denote coordinates on $\Pi_{l}^{+}$and $\Pi_{l}^{-}$as $\left(x_{0 l}, y_{0 l}\right)$ and $\left(x_{1 l}, y_{1 l}\right)$, respectively. Then, the global maps can be written as follows. Map $T_{12}(\mu)$ has the following form (the Taylor expansion near the point $\left.x_{11}=0, y_{11}=y_{1}^{-}(\mu)\right)$

$$
\begin{aligned}
& \bar{x}_{02}-x_{2}^{+}(\mu)=a_{12}(\mu) x_{11}+b_{12}(\mu)\left(y_{11}-y_{1}^{-}(\mu)\right)+\ldots \\
& \bar{y}_{02}=c_{12}(\mu) x_{11}+d_{12}(\mu)\left(y_{11}-y_{1}^{-}(\mu)\right)+\ldots
\end{aligned}
$$


where coefficients $a_{12}, \ldots, d_{12}$ depend on $\mu$, in general; $x_{2}^{+}(0)=x_{2}^{+}, y_{1}^{-}(0)=y_{1}^{-}$. Note that points $\left(x_{2}^{+}(\mu), 0\right)$ and $\left(0, y_{1}^{-}(\mu)\right)$ are the intersection points of the orbit $\Gamma_{12}(\mu)$ with $\Pi_{2}^{+}$and $\Pi_{1}^{-}$, where by transversality of the intersection of stable and unstable manifolds $d_{12}(0) \neq 0$ and $x_{2}^{+}(\mu)=y_{1}^{-}(\mu)$ for all $\mu$. Moreover, the Jacobian $J_{12} \equiv a_{12} d_{12}-b_{12} c_{12}$ of the map $T_{12}$ is calculated in the point $M_{1}^{-}$at $\mu=0$ is nonzero since $T_{12}$ is a diffeomorphism.

It should be noted that reversibility imposes some conditions on the expressions in the right hand side of (5). Namely, consider the map $T_{12}: \Pi_{1}^{-} \rightarrow \Pi_{2}^{+}$, where $\Pi_{1}^{-} \in U_{1}, \Pi_{2}^{+} \in U_{2}$. In local coordinates, we have

$$
T_{12}=\tilde{q}^{-1} \circ f^{n_{2}} \circ q=R_{12} \circ q^{-1} \circ R^{-1} \circ f^{n_{2}} \circ q .
$$

It is easy to check that

$$
R_{12}^{-1} \circ T_{12} \circ R_{12}^{-1}=T_{12}^{-1} .
$$

In order to simplify we rewrite formulas of (5) in the form

$$
\begin{aligned}
& \left.\bar{x}_{02}-x_{2}^{+}(\mu)=F\left(x_{11}, y_{11}-y_{1}^{-}(\mu), \mu\right)\right), \\
& \left.\bar{y}_{02}=G\left(x_{11}, y_{11}-y_{1}^{-}(\mu), \mu\right)\right),
\end{aligned}
$$

It follows from (6) and uniqueness $x_{2}^{+}(\mu)=y_{1}^{-}(\mu)$ that $T_{12}^{-1}$ has the following form

$$
\begin{aligned}
& \left.x_{11}=G\left(\bar{y}_{02}, \bar{x}_{02}-x_{2}^{+}(\mu), \mu\right)\right), \\
& \left.y_{11}-y_{1}^{-}(\mu)=F\left(\bar{y}_{02}, \bar{x}_{02}-x_{2}^{+}(\mu), \mu\right)\right) .
\end{aligned}
$$

Let us substitute the expressions for $\bar{x}_{02}-x_{2}^{+}(\mu)$ and $\bar{y}_{02}$ given by (7) into right side of (8). We obtain uniqueness

$$
\begin{aligned}
& \left.\left.\left.x_{11}=G\left(G\left(x_{11}, y_{11}-y_{1}^{-}(\mu), \mu\right)\right), F\left(x_{11}, y_{11}-y_{1}^{-}(\mu), \mu\right)\right), \mu\right)\right), \\
& \left.\left.\left.y_{11}-y_{1}^{-}(\mu)=F\left(G\left(x_{11}, y_{11}-y_{1}^{-}(\mu), \mu\right)\right), F\left(x_{11}, y_{11}-y_{1}^{-}(\mu), \mu\right)\right), \mu\right)\right) .
\end{aligned}
$$

or

$$
\begin{aligned}
& x_{11}=\left(c_{12}^{2}+a_{12} d_{12}\right) x_{11}+d_{12}\left(b_{12}+c_{12}\right)\left(y_{11}-y_{1}^{-}\right)+\ldots, \\
& y_{11}-y_{1}^{-}=a_{12}\left(b_{12}+c_{12}\right) x_{11}+\left(b_{12}^{2}+a_{12} d_{12}\right)\left(y_{11}-y_{1}^{-}\right)+\ldots
\end{aligned}
$$

From this and $d_{12} \neq 0$, it follows that

$$
b_{12}(\mu)=-c_{12}(\mu), \quad a_{12}(\mu) d_{12}(\mu)+b_{12}^{2}(\mu)=1 .
$$

Of course, there exist special relations on coefficients of high order terms also.

The following lemma describes how the reversibility influences the form of the global map.

Lemma 1 The map $T_{12}$ can be written implicitly (in cross coordinates) as

$$
\begin{aligned}
& \bar{x}_{02}-x_{2}^{+}(\mu)=\Phi\left(x_{11}, \bar{y}_{02}, \mu\right), \\
& y_{11}-y_{1}^{-}=\Phi\left(\bar{y}_{02}, x_{11}, \mu\right) .
\end{aligned}
$$

where

$$
\Phi(u, v, \mu)=A(\mu) u+B(\mu) v+O\left(u^{2}+|u v|+v^{2}\right), \quad B(\mu) \neq 0
$$

Proof Note that the equation $w=G(u, v, \mu)$ define variable $v$ uniquely as functions of $u$ and $v$, i.e. $v=\Phi(u, w)$, because $\frac{\partial G}{\partial v}(0,0, \mu) \neq 0$. It follows from the second equation of the system (7) and the first equation of (8) that $\bar{x}_{02}-x_{2}^{+}(\mu)=\Phi\left(x_{11}, \bar{y}_{02}, \mu\right)$ and $y_{11}-y_{1}^{-}=\Phi\left(\bar{y}_{02}, x_{11}, \mu\right)$. It is easy to check that $\Phi(u, v, \mu)=A(\mu) u+B(\mu) v+O\left(u^{2}+|u v|+v^{2}\right)$, where $B(\mu) \neq 0$.

The map $T_{21}(\mu) \equiv f_{\mu}^{n_{2}}: \Pi_{2}^{-} \rightarrow U_{1}$ can be written in the following form (the Taylor expansion near the point $\left.x_{12}=0, y_{12}=y_{2}^{-}(\mu)\right)$

$$
\begin{aligned}
& \bar{x}_{01}-x_{1}^{+}(\mu)=E(\mu)+a_{21}(\mu) x_{12}+b_{21}(\mu)\left(y_{12}-y_{2}^{-}(\mu)\right) \ldots \\
& \bar{y}_{01}=\mu+c_{21}(\mu) x_{12}+d_{21}(\mu)\left(y_{12}-y_{2}^{-}(\mu)\right)^{2}+\ldots
\end{aligned}
$$


We now note that ( $R$-equivariant) coordinate transformations that preserve the $R$-reversibility of the map, automatically preserve the relation $x_{1}^{+}(\mu)=y_{2}^{-}(\mu)$. We now may perform such a coordinate transformation so that $y_{2}^{-}(\mu)$ is such that the second equation of (14) does not contain a term linear in $y_{12}$, even when $\mu \neq 0$. However, unlike the situation in the nonreversible case $[13,20,12]$, we will be left with a term $E(\mu)$ in the first equation of (14), which is generally nonzero if $\mu \neq 0$. We furthermore note that $d_{21}(0) \neq 0$ since $W^{u}\left(O_{2}\right)$ and $W^{s}\left(O_{1}\right)$ have a quadratic tangency at point $M_{1}^{+}$and $J_{21} \equiv-b_{21}(0) c_{21}(0) \neq 0$ since $T_{21}$ is a diffeomorphism.

Note that $\mu$ enters in the second equation of (14) as a constant term that serves as the splitting parameter of the manifolds $W^{u}\left(O_{2}(\mu)\right)$ and $W^{s}\left(O_{1}(\mu)\right)$ at the point $M_{1}^{+}$.

It follows from reversibility that

$$
R_{12} \circ T_{21} \circ R_{12}=T_{21}^{-1} .
$$

Hence, $T_{21}^{-1}$ can be written as

$$
\begin{aligned}
& y_{12}-y_{2}^{-}(\mu)=E(\mu)+a_{21}(\mu) \bar{y}_{01}+b_{21}(\mu)\left(\bar{x}_{01}-x_{1}^{+}\right) \ldots \\
& x_{12}=\mu+c_{21}(\mu) \bar{y}_{01}+d_{21}(\mu)\left(\bar{x}_{01}-x_{1}^{+}\right)^{2}+\ldots
\end{aligned}
$$

Like we did before for global map $T_{12}$ (see formulaes (9) and (10)), we obtain the relations on coefficients of $T_{21}$. It is furthermore easy to check that

$$
\begin{gathered}
E(\mu)=-a \mu / 2+O\left(\mu^{2}\right) ; \quad a_{21}(\mu)=a+a_{\mu} \mu \\
b_{21}(\mu)=1+b_{\mu} \mu ; \quad c_{21}(\mu)=-1+c_{\mu} \mu
\end{gathered}
$$

For sufficiently large $k$ and small $\mu$ we define the map $T_{01}^{k}(\mu): \Pi_{1}^{+} \rightarrow \Pi_{2}^{-}$. It can be written in the form $[8,9]$

$$
\begin{aligned}
& x_{11}=\lambda(\mu)^{k} x_{01}\left(1+\hat{\gamma}^{-k} p_{k}\left(x_{01}, y_{11}, \mu\right)\right) \\
& y_{01}=\gamma(\mu)^{-k} y_{11}\left(1+\hat{\gamma}^{-k} q_{k}\left(x_{01}, y_{11}, \mu\right)\right)
\end{aligned}
$$

where $\hat{\gamma}^{-1}=\max \left\{\gamma^{-1}, \lambda\right\}$, and the functions $p_{k}$ and $q_{k}$ are uniformly bounded in $k$ along with all derivatives up to order $(r-2)$. Thus, in these coordinates, for large $k$ the map $T_{01}^{k}$ will be asymptotically close to a linear map.

We recall from (3) that

$$
T_{01}^{k}=R_{12}^{-1} \circ T_{02}^{-k} \circ R_{12} .
$$

Hence, the map $T_{02}^{-k}$ can be written in the form

$$
\begin{aligned}
& y_{02}=\lambda(\mu)^{k} y_{12}\left(1+\hat{\gamma}^{-k} p_{k}\left(y_{12}, x_{02}, \mu\right)\right) \\
& x_{12}=\gamma(\mu)^{-k} x_{02}\left(1+\hat{\gamma}^{-k} q_{k}\left(y_{12}, x_{02}, \mu\right)\right)
\end{aligned}
$$

\subsection{Geometric Tools}

\section{Strips and Horseshoes.}

We recall that $U$ is a small neighborhood of $C=\left\{O_{1}, O_{2}, \Gamma_{12}, \Gamma_{21}\right\}$ and $U$ consist of two small discs $U_{1}$ and $U_{2}$ containing points $O_{1}$ and $O_{2}$, and a finite number of small discs surrounding the points of orbits $\Gamma_{12}$ and $\Gamma_{21}$ which are located outside $U_{1}$ and $U_{2}$ (Fig. 2). The subject of our study is the set $N(\mu)$ of orbits of the map $f(\mu)$ that lie entirely in $U$.

Consider the neighbourhood $U_{l}, l=1,2$. Note that orbits of $N$ must intersect the neighbourhoods $\Pi_{l}^{+}$ and $\Pi_{l}^{-}$(otherwise, these orbits would be far from $\Gamma$ ). However, not all orbits that start in $\Pi_{l}^{+}$arrive in $\Pi_{l}^{-}$. The set of the points whose orbits get into $\Pi_{l}^{-}$fills a countable number of strips $\sigma_{k}^{0 l}=\Pi_{l}^{+} \cap T_{0 l}^{-k} \Pi_{l}^{-}$ which accumulate on $W_{\mathrm{loc}}^{s}\left(O_{l}\right)$. The way of constructing these strips is obvious from Fig. 3a. In turn, the images of the strips $\sigma_{k}^{0 l}$ under the maps $T_{0 l}^{k}$ give on $\Pi_{l}^{-}$a sequence of strips $\sigma_{k}^{1 l}$ which accumulate on $W_{\text {loc }}^{u}\left(O_{l}\right)$ (Fig. 3b). This follows from the local representations of $T_{0 l}^{k}(18)$. 
a)

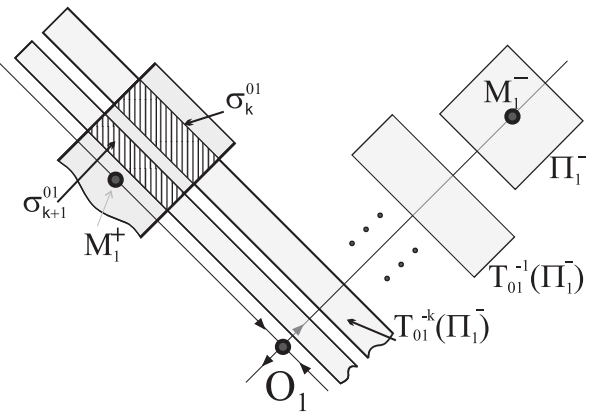

b)

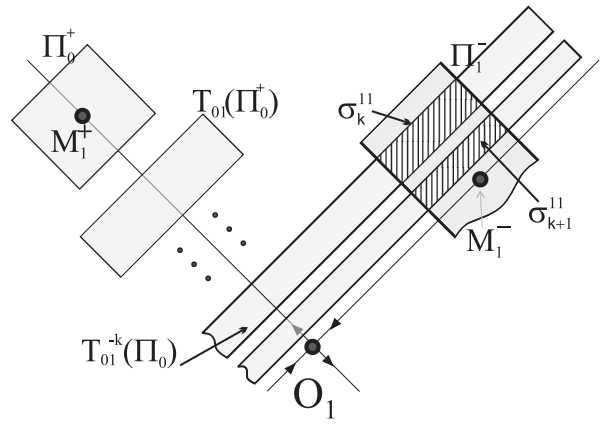

Figure 3: Method of a construction of the strips $\sigma_{k}^{01}$ and $\sigma_{k}^{11}$

Neighborhoods $\Pi_{l}^{+}$and $\Pi_{l}^{-}$may be taken so that to contain all the strips $\sigma_{k}^{0 l}$ and $\sigma_{k}^{1 l}$ with numbers $k \geq \bar{k}$, for some $\bar{k}$, and not to intersect with $\sigma_{k}^{0 l}$ and $\sigma_{k}^{1 l}$ for all $k<\bar{k}$. Obviously, if $\operatorname{diam} \Pi_{l}^{+} \cdot \operatorname{diam} \Pi_{l}^{-} \rightarrow 0$, then $\bar{k} \rightarrow \infty$.

The images $T_{12} \sigma_{k}^{11}$ of the strips $\sigma_{k}^{11}$ are strips accumulated at $T_{12} W_{\text {loc }}^{u}\left(O_{1}\right)$ as $k \rightarrow \infty$ (Fig. 4 ). It is clear that orbits of $N$ must intersect $\Pi_{2}^{+}$in points lying in intersections of strips $T_{12} \sigma_{i}^{11}$ and strips $\sigma_{j}^{02}$ for $i, j \geq \bar{k}$.

Evaluating the action of the global map $T_{21}$, we note that the images $T_{21} \sigma_{k}^{12}$ of the strips $\sigma_{k}^{12}$ have the shape of horse-shoes accumulating to $T_{21} W_{\text {loc }}^{u}\left(O_{2}\right)$ as $k \rightarrow \infty$ (Fig. 4). Of course, orbits of $N$ must intersect $\Pi_{1}^{+}$in points lying in intersections of horse-shoes $T_{21} \sigma_{i}^{12}$ and strips $\sigma_{j}^{01}$ for $i, j \geq \bar{k}$. Hence, the structure of $N$ depends essentially on the geometrical properties of such intersections.

\section{The Types of Intersections of the Strips and Horseshoes.}

The horse-shoe $T_{21} \sigma_{i}^{21}$ can have different types of intersections with the strips $\sigma_{j}^{01}$. We call the intersection regular if the set $T_{21} \sigma_{i}^{12} \cap \sigma_{j}^{01}$ is non-empty and consists of two connected components $\sigma_{j i}^{01}$ and $\sigma_{j i}^{02}$. The different types of intersections are illustrated in Fig. 5: the horse-shoe has a regular intersection with the strip $\sigma_{j}^{01}$, an irregular intersection with the strip $\sigma_{k}^{01}$ and empty intersection with the strip $\sigma_{l}^{01}$.

In [20], Gonchenko and Shilnikov established conditions for regular and irregular intersections of the strips and horseshoes. We may adapt these conditions to our case, taking into account the reversibility $\left(\right.$ eg $\left.c_{21}=-1, \ldots.\right)$.

Recall that $d_{21} \neq 0$ is the coefficient in (14) establishing the quadratic tangency. The sign of $d_{21}$ signals whether the tangency is from "above" or "below", with respect to the stable and unstable manifolds (see also Fig. 1).

If the inequality

$$
d_{21}\left(\gamma^{-i} x_{2}^{+}-\mu+\gamma^{-j} x_{2}^{+}\right)>S_{i j}(\bar{k}),
$$

is satisfied, where $S_{i j}=S_{1}\left(|\gamma|^{-i}+|\gamma|^{-j}\right)\left(|\lambda|^{\bar{k}}+|\gamma|^{-\bar{k}}\right)$ and $S_{1}$ is some positive constant independent from $i, j$ and $\bar{k}$, then the intersection of $T_{21}(\mu)\left(\sigma_{j}^{12}\right)$ and the strip $\sigma_{i}^{01}$ is regular.

The inequality

$$
d_{21}\left(\gamma^{-i} x_{2}^{+}-\mu+\gamma^{-j} x_{2}^{+}\right)<-S_{i j}(\bar{k})
$$

is a sufficient condition for an intersection of $T_{21}(\mu)\left(\sigma_{j}^{12}\right)$ and $\sigma_{i}^{01}$ to be empty.

It is clear from (21) and (22) that the inequality

$$
\left|d_{21}\left(\gamma^{-i} x_{2}^{+}-\mu+\gamma^{-j} x_{2}^{+}\right)\right| \leq S_{i j}(\bar{k})
$$

is necessary in order for the horse-shoe $T_{21}(\mu)\left(\sigma_{j}^{12}\right)$ to have an irregular intersection with the strip $\sigma_{i}^{01}$.

Inequalities (21)-(23) have quite a simple geometrical interpretation, see Fig. 6: The strip $\sigma_{j}^{12}$ is a thin rectangle with the central line $y_{12}=\gamma^{-j} x_{2}^{+}$. The strip $\sigma_{i}^{01}$ is a thin rectangle with the central line $x_{01}=$ $\gamma^{-i} x_{2}^{+}$. The strip $\sigma_{j}^{01}$ is mapped by the map $T_{21}$ onto a horse-shoe with the parabola $y_{01}=\mu-\gamma^{-j} x_{2}^{+}+$ 


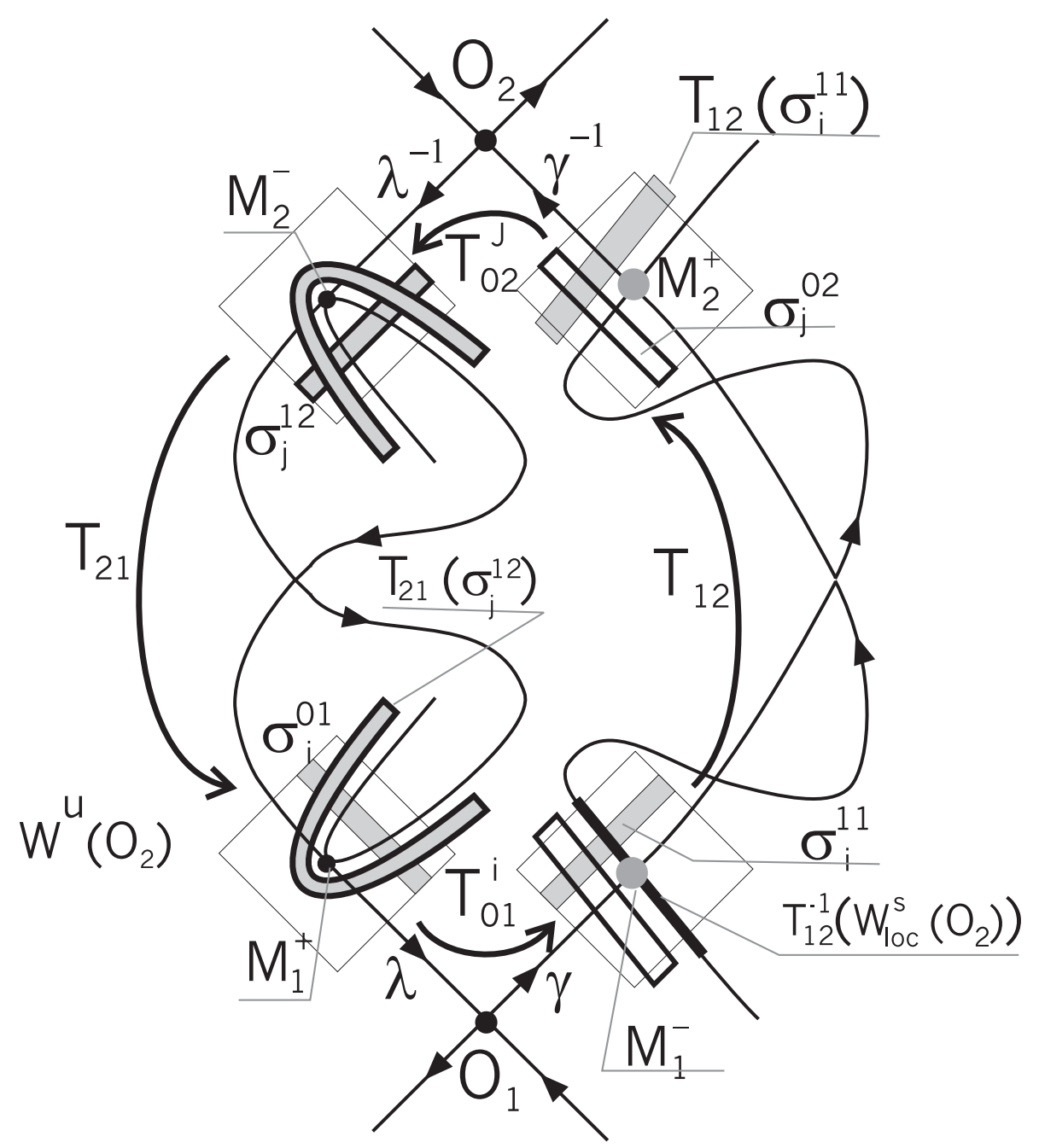

Figure 4:

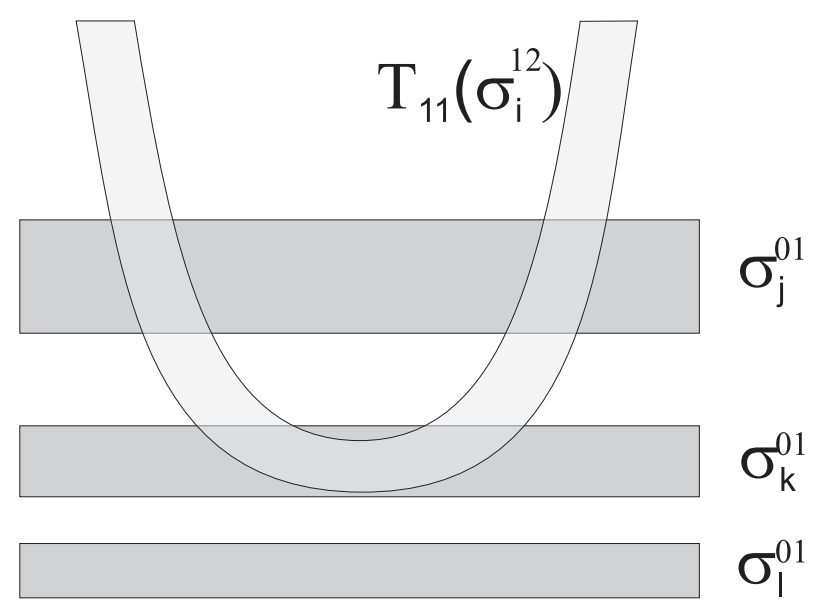

Figure 5: Different types of intersection of strips and horseshoes 


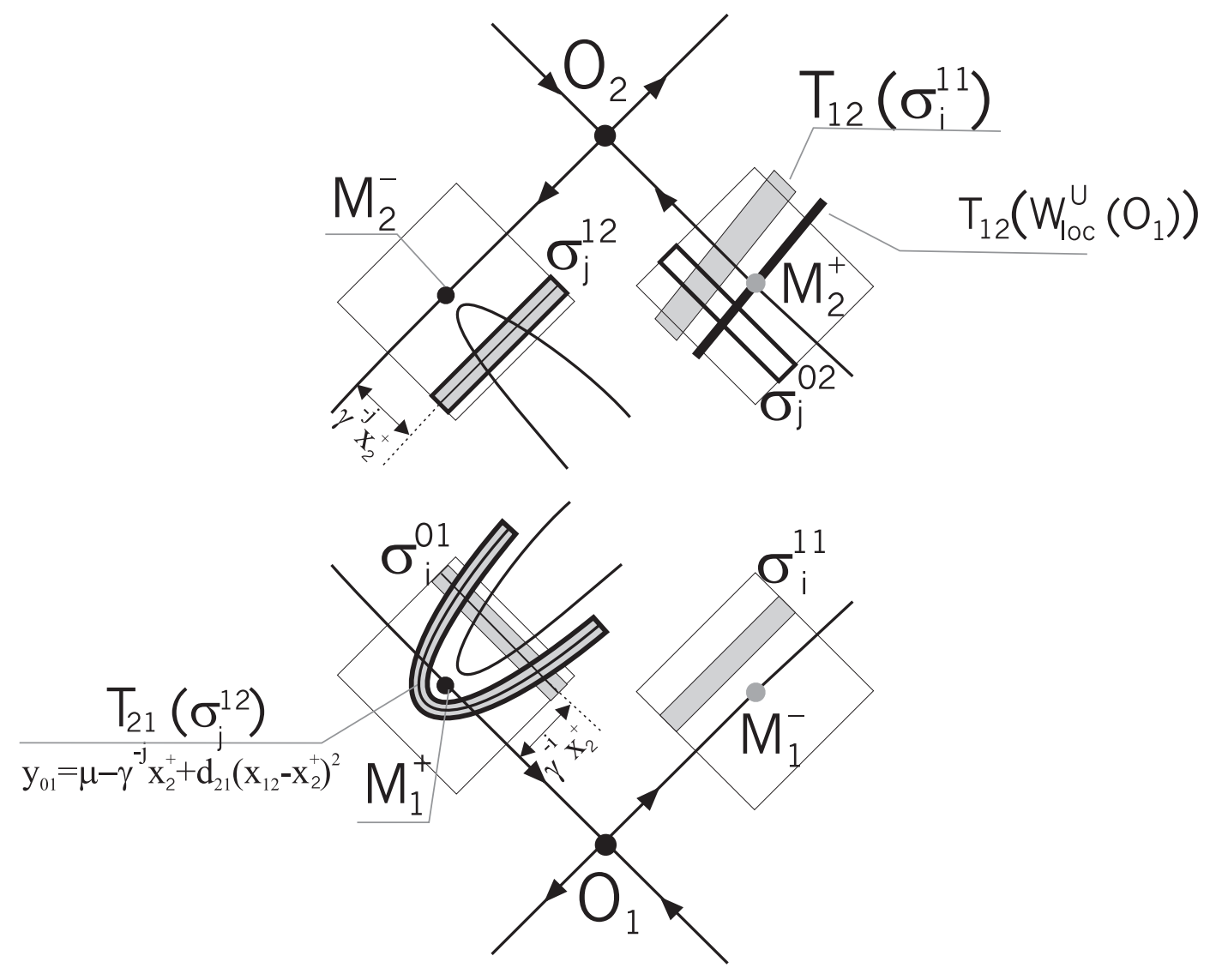

Figure 6: 


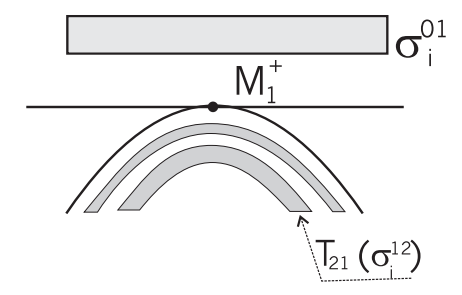

a) $d_{21}<0, \lambda>0, \gamma>0$

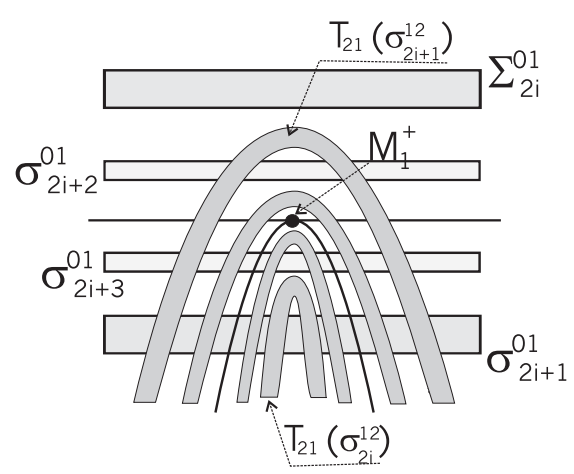

c) $d_{21}<0, \lambda<0, \gamma<0$

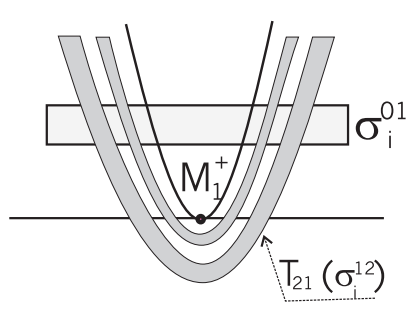

b) $d_{21}>0, \lambda>0, \gamma>0$

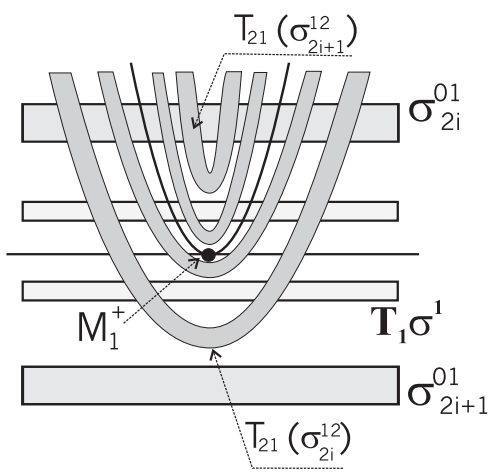

d) $\mathrm{d}_{21}>0, \lambda<0, \gamma<0$

Figure 7:

$d_{21}\left(\left(x_{12}-x_{2}^{+}\right)\right)^{2}$ as a central line. The condition $d_{21}\left(\gamma^{-i} x_{2}^{+}-\mu+\gamma^{-j} x_{2}^{+}\right)>0$ means that the straight line $y_{12}=\gamma^{-j} x_{2}^{+}$and the parabola intersect in two points, and the condition $d_{21}\left(\gamma^{-i} x_{2}^{+}-\mu+\gamma^{-j} x_{2}^{+}\right)<0$ means that they have an empty intersection. The coefficient $S_{i j}(\bar{k})$ in $(21)-(23)$ is due to the non-zero thicknesses of the strip and horse-shoe.

\subsection{Dynamics near heteroclinic cycles}

We consider the diffeomorphism $f_{0}$, with initial non-transversal symmetric heteroclinic cycle.

It is well know that the properties of orbits in $N$ essentially depend on the character of the intersection of strips and horseshoes. In the case when all strips and all horse-shoes intersect regularly, it can be proven $[7,9,20]$ that all orbits from $N \backslash \Gamma_{21}$ are of saddle type. Moreover, $N$ admits a full description in terms of symbolic dynamics: the orbits of $N$ are in one-to-one correspondence with orbits of a subsystem of the topological Bernoulli shift on four symbols $\{1,2,3,4\}$. Hence, an analysis of the structure of integer solutions of inequalities (21)-(23) is an essential part of the study of orbits of the set $N$. The sets of such solutions obviously depend on the signs of parameters $\lambda, \gamma$ and $d_{21}$. Geometrically, this is related to the fact that the signs of these values determine the character of the reciprocal position of the manifolds $W_{\text {loc }}^{s}\left(O_{1}\right)$ and $T_{1} W_{\text {loc }}^{u}\left(O_{2}\right)$ in a neighborhood of the heteroclinic point $M_{1}^{+}$.

We now consider the cases of positive and negative multipliers, separately.

The case of positive $\lambda$ and $\gamma$. The different possible cases here depend on the signs of $d_{21}$ and types of reversibility are shown in Fig. 1. It follows from (22), when $d_{21}<0$ and $\lambda>0$ and $\gamma>0$, that the horse-shoes $T_{21} \sigma_{i}^{12}$ and the strips $\sigma_{j}^{01}$ do not intersect, since they lie at opposite sides of $W_{\text {loc }}^{s}\left(O_{1}\right)$, see Fig. 7a. It is obvious that in this case the set $N$ has a trivial structure: $N=\left\{O_{1}, O_{2}, \Gamma_{12}, \Gamma_{21}\right\}$. In terms of the classification given by Gonchenko, Turaev \& Shilnikov [7, 20], the diffeomorphisms under consideration are called diffeomorphisms of the first class.

In case $d_{21}>0$, evidently, inequality (21) holds for sufficiently large $i$ and $j$ : the intersection of $T_{21} \sigma_{i}^{12}$ with $\sigma_{j}^{01}$ is regular for any $i, j \geq \bar{k}$ (Fig. 7). In this case all orbits from $N \backslash \Gamma$ are of saddle 


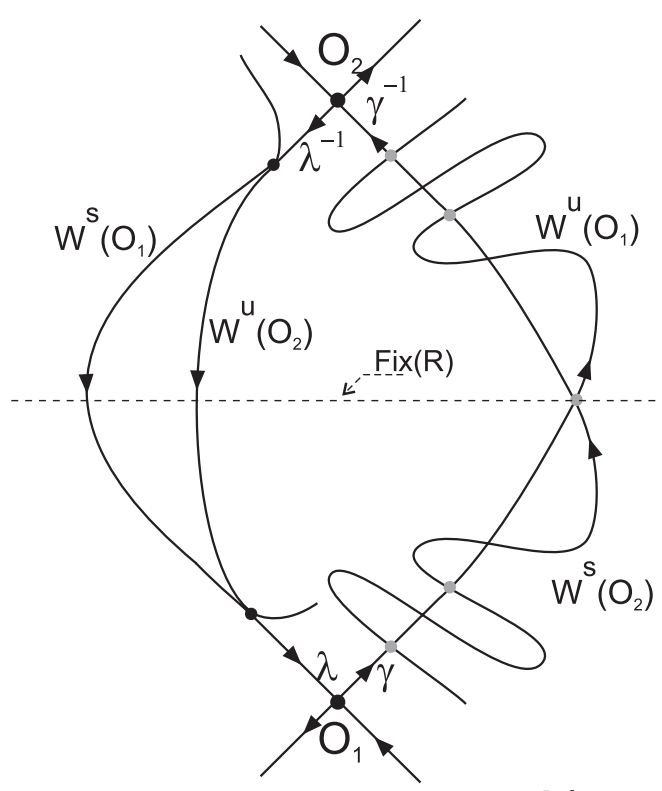

A)

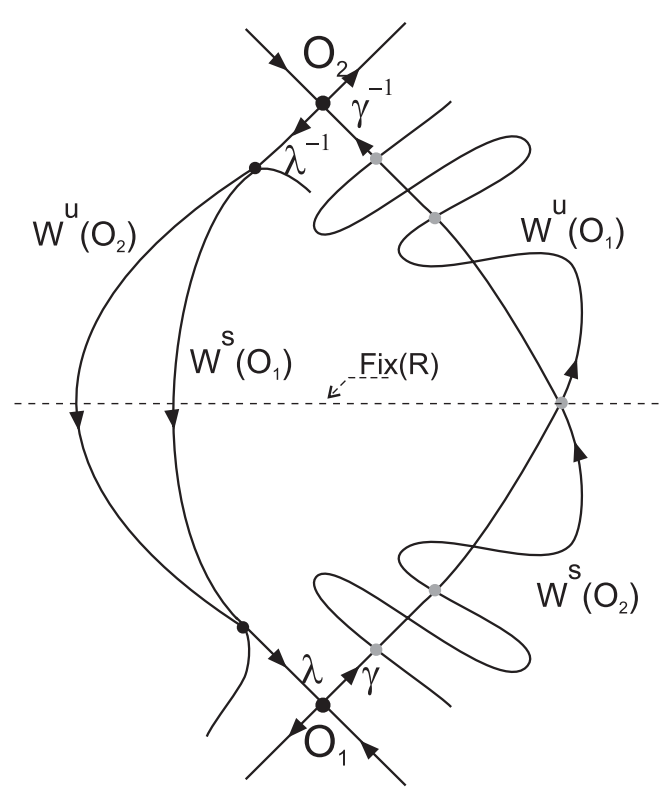

B)

Figure 8: A priori non-symmetric heteroclinic cycles

type. The set $N$ is a non-uniformly hyperbolic set and admits a full description in terms of symbolic dynamics [20]. According to [20] such diffeomorphisms belong to the second class of diffeomorphisms with a non-transversal heteroclinic cycle.

Remark 1 In [20] it has been shown that in general diffeomorphisms, there is also a third class of diffeomorhisms with non-transverse heteroclinic cycle, where the set $N_{0}$, in general, does not admit a full description and contains a nontrivial hyperbolic subset. Moreover, on the bifurcation surface of these diffeomorphisms there is a dense set of diffeomorphisms with structurally unstable periodic and homoclinic orbits (with any order of degeneracy). For the case of positive multipliers of saddle fixed points, diffeomorphisms with the above-mentioned heteroclinic cycle can belong to the third class only if $c_{21}>0$.

It is important to realize that we consider a symmetric heteroclinic cycle. We proved before that due to the reversibility, the coefficient $c_{21}$ is always negative $\left(c_{21}=-1\right)$. This implies that in the case of positive multipliers of saddle fixed points, there are no diffeomorphisms of the third class [20].

In turn, this means that a non-transversal heteroclinic orbit of $f$ with the geometry of manifolds as sketched in Fig. 8 cannot be symmetric with respect to the reversing symmetry $R$. We say, in this case, that $f$ has an a priori non-symmetric heteroclinic cycle.

The case of negative $\lambda$ and $\gamma$. In this case $f$ is a diffeomorphism of the third class (Fig. 7c, Fig. 7d). But there is a principal difference with the non-reversible case. Namely, it follows from the inequalities (21 -23) (at $\mu=0)$ that the intersection of strips and horseshoes cannot be irregular, so that the set $N \backslash \Gamma_{21}$ contains only saddle type orbits. Again the set $N \backslash \Gamma_{21}$ is non-uniformly hyperbolic and is topologically conjugated to a subsystem of a Bernoulli shift. Of course, such type of diffeomorphisms are not typical for non-reversible diffeomorphisms.

\section{Newhouse regions for homoclinic and heteroclinic tangencies}

In this section we prove the first part of Theorem 1, concerning the density of diffeomorphisms with non-transversal homoclinic and heteroclinic cycles in parameter intervals. It is worth mentioning that conceptually, the proof is close to results in [20]. 


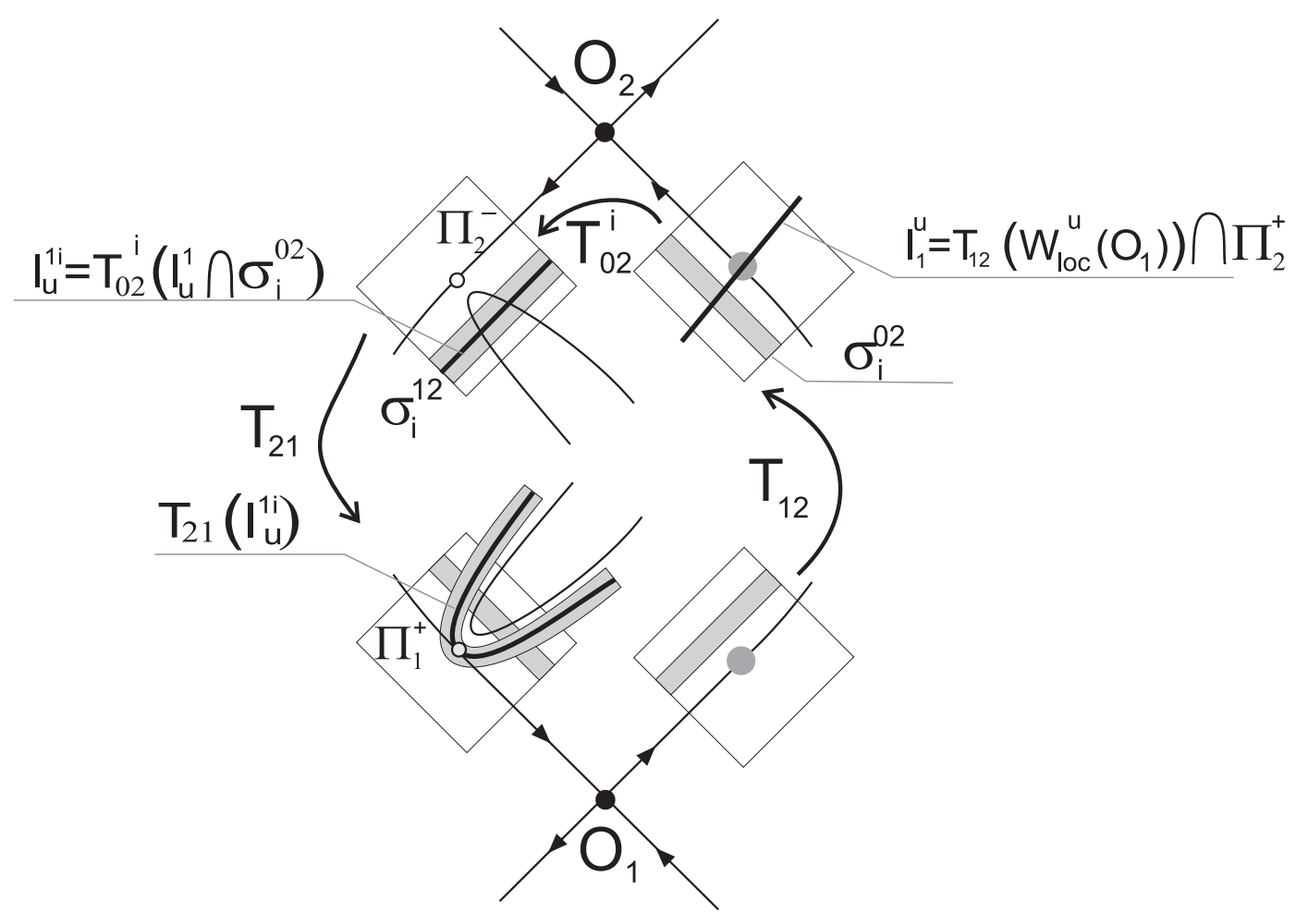

Figure 9:

Recall that in case $\lambda>0$ and $\gamma>0$, if $\mu \leq 0$ the diffeomorphism $f_{\mu}$ has trivial dynamics for $d_{21}<0$, and hyperbolic behaviour for $d_{21}>0$ ( in this case the nonwandering set $N(\mu)$ admits full description in the terms of symbolic dynamics). Therefore, Newhouse regions can appear only for positive $\mu$. In case $\lambda>0, \gamma<0$, generally speaking, dynamics of $f_{\mu}$ is non-uniformly hyperbolic only if $\mu=0$ and Newhouse regions arise both when $\mu>0$ and $\mu<0$. Here, we will discuss in detail only the case $\mu>0$, although the proofs can be carried through for negative values of $\mu$.

From now on we assume that $\mu \geq 0$.

\subsection{Density of diffeomorphisms with homoclinic tangencies}

Lemma 2 There exists a sequence $\left\{\mu_{i}\right\}$ of parameter values $\mu$ such that $\mu_{i} \rightarrow 0$ as $i \rightarrow \infty, \mu_{i}>0$, and the diffeomorphism $f_{\mu_{i}}$ has a structurally unstable single-round orbit $\Gamma_{1 i}$ homoclinic to $O_{1} \cdot{ }^{1}$, such that the tangency of the manifolds $W^{s}\left(O_{1}\right)$ and $W^{u}\left(O_{2}\right)$ along $\Gamma_{1 i}$ is quadratic and, for $\mu=\mu_{i}, f_{\mu}$ is a transversal family which unfolds the non-transversal homoclinic orbit $\Gamma_{1 i}$ generically.

\section{Proof}

The idea is that we iterate a segment of unstable manifolds of $O_{1}$ to obtain a segment of the same manifolds in the neighbourhood $\Pi_{1}^{+}$(Fig. 9). We denote as $l_{u}^{1}(\mu)$ the segment $T_{12}\left(W_{\text {loc }}^{u}\left(O_{1}\right)\right) \cap \Pi_{2}^{+}$of the unstable manifold of the point $O_{1}$. For small $\mu$, the segment $l_{u}^{1}(\mu)$ intersects the manifold $W_{\text {loc }}^{s}\left(O_{2}\right)$ transversally. It is easy to see from (20) that the curves $l_{u}^{1 i} \equiv T_{02}^{i}\left(l_{u}^{1} \cap \sigma_{i}^{02}\right)$ on $\Pi_{2}^{-}$accumulate to the segment $l_{u}^{2} \equiv W_{\text {loc }}^{u}\left(O_{2}\right) \cap \Pi_{2}^{-}$(Fig. 9), which is defined by the equation $x_{12}=0$ on $\Pi_{2}^{-}$. In this case, by the virtue of (20) and (5), the equation of the curve $l_{u}^{1 i}$ is $x_{12}=\gamma(\mu)^{-i} x_{2}^{+}(\mu)(1+\ldots)$ and, by virtue of (14), for $\mu=\mu_{i} \equiv \gamma^{-i} x_{2}^{+}(1+\ldots)$ the diffeomorphism $f_{\mu}$ has in $U$ a single-round non-transversal homoclinic orbit $\Gamma_{1 i}$ of the point $O_{1}$. For large $i$, the tangency of the stable and the unstable manifold of the point $O_{1}$ at the points of the orbit $\Gamma_{1 i}$ is quadratic, and the family $f_{\mu}$ is transversal to the bifurcation surface

\footnotetext{
${ }^{1}$ For a definition of single-round orbits, see Section 4.
} 
of diffeomorphisms with a non-transversal homoclinic orbit which is close to $\Gamma_{1 i}$.

Remark 2 It follows from reversibility that $f_{\mu_{i}}$ has a homoclinic tangency also at the point $\mathrm{O}_{2}$. Indeed, $f_{\mu_{i}}$ has a structurally unstable single-round homoclinic orbit $\Gamma_{2 i}$ which is the image of $\Gamma_{1 i}$ under $R$.

Lemma 3 There exists a sequence of intervals $\left\{\delta_{i}\right\}$ of values of the parameter $\mu$ which accumulate at $\mu=0$ such that parameter values $\mu$ at which the family $f_{\mu}$ unfolds generically the homoclinic tangency of the point $O_{1}\left(\right.$ and $O_{2}$ ) are dense in the intervals $\delta_{i}$.

Proof This lemma follows directly from the Newhouse theorem [26], lemma 2 and remark 2.

\subsection{Density of diffeomorphisms with symmetric heteroclinic cycles}

Since $\Gamma_{21}=R\left(\Gamma_{21}\right)$, it is straightforward to prove that there exist a point $q, q \in \Gamma_{21}$, such that $q \in$ $\operatorname{Fix}(R) \cup \operatorname{Fix}(R \circ f)$. By our assumptions, the sets $\operatorname{Fix}(R)$ and $\operatorname{Fix}(R \circ f)$ are one-dimensional curves. Moreover, it can be shown that the stable manifold $W^{s}$ of $O_{1}$ and the unstable manifold $W^{u}$ of $O_{2}$ have a quadratic tangency with the set $\operatorname{Fix}(R)$ from opposite directions at the same point $q$. We recall that there exists a positive integer $n_{2}$ such that $f_{0}^{n_{2}}\left(M_{2}^{-}\right)=M_{1}^{+}$. If $n_{2}$ is even then $q \in \operatorname{Fix}(R)$. For odd $n_{2}$, the point $q$ belongs to $\operatorname{Fix}(R \circ f)$. We now suppose that $q \in \operatorname{Fix}(R)$. (The proof is analogous if $q \in \operatorname{Fix}(R \circ f)$.) Let $s_{1}(\mu)$ denote the segment $f_{\mu}^{n_{2} / 2}(\operatorname{Fix}(R)) \cap \Pi_{1}^{+}$. The curve $s_{1}(\mu)$ has a tangency with $W_{\text {loc }}^{s}\left(O_{1}\right)$ at the point $M_{2}^{-}$.

Proposition 1 For any $\mu \in\left(0, \mu_{0}\right)$ and sufficiently large $j$ (independent of $\mu$ ) there exist constants $l$, $l=l(i, j)$ such that $f^{l}(\operatorname{Fix}(R))$ accumulate to $W_{\mathrm{loc}}^{u}\left(O_{1}\right)$ in $\Pi_{1}^{-}$and the distance between $f^{l}(\mathrm{Fix}(R))$ and $W_{\mathrm{loc}}^{u}\left(O_{1}\right)$ is approximately equal to $x_{1}^{+} \lambda^{j}$.

Proof Let us choose some $\mu$ inside the interval $\left(0, \mu_{0}\right)$. We discuss the cases $d_{21}<0$ and $d_{21}>0$ separately.

$d_{21}<0$ : In this case we have a simplest construction of the proof. Indeed, for $\mu=0$ in neighborhood $\Pi_{1}^{+}$there exist a tangency of $W_{\text {loc }}^{s}\left(O_{1}\right), W_{\text {loc }}^{u}\left(O_{2}\right)$ and the curve $s_{1}(\mu)$ at the point $M_{1}^{+}$(Fig. 10a). We can split this tangency. Let us choose the parameter $\mu$ from the interval $\left(0, \mu_{0}\right)$. We obtain two new transversal heteroclinic points $M_{2 a}^{-}$and $M_{2 b}^{-}$. The last belong to new symmetric heteroclinic orbits, therefore $s_{1}(\mu)$ must intersect $W_{u}\left(O_{2}\right)$ transversally in $M_{2 a}^{-}$and $M_{2 b}^{-}$also (Fig. 10b). As a consequence, for sufficiently large $j(j>\tilde{k}(\mu))$ the curve $s_{1}(\mu)$ intersects the strip $\sigma_{j}^{01}$ along two components $s_{j 1}^{01}$ and $s_{j 2}^{01}$ (Fig. 10). By the virtue of (20), as $j \rightarrow \infty$, the curves $s_{j 1}^{11} \equiv T_{01}^{j}\left(s_{j 1}^{01}\right)$ on $\Pi_{1}^{-}$accumulate to the segment $l_{u}^{1} \equiv W_{\text {loc }}^{u}\left(O_{1}\right) \cap \Pi_{1}^{-}$, which is defined by the equation $x_{11}=0$ on $\Pi_{1}^{-}$. It follows from (20) that the distance between $s_{j 1}^{11}$ and $W_{\text {loc }}^{u}\left(O_{1}\right)$ is approximately equal to $x_{1}^{+} \lambda^{j}$. By construction, $s_{j 1}^{11}=f^{l}(\operatorname{Fix}(R)) \cap \Pi_{1}^{-}$, where $l=n_{2} / 2+j$.

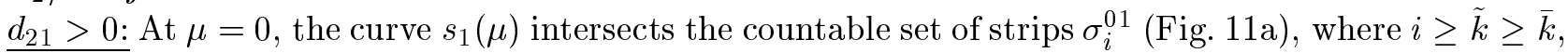
$\tilde{k}$ is some integer. Hence, there exist $\mu_{0}$ and $k=k\left(\mu_{0}\right)$ such that the curve $s_{1}(\mu)$ intersects $\sigma_{k}^{01}$ along two components $s_{k 1}^{01}$ and $s_{k 2}^{01}$ for all $\mu \in\left(0, \mu_{0}\right)$, see Fig. 11b. It should be note that in this case the constant $k$ depends on $\mu$ and we can not assume $j=k$ (as in the proof when $d_{21}<0$ ). By virtue of (20), for large $k$ (and small $\mu$ ) the curves $s_{k 1}^{11} \equiv T_{01}^{k}\left(s_{k 1}^{01}\right)$ on $\Pi_{1}^{-}$accumulate to the segment $l_{u}^{1} \equiv W_{\text {loc }}^{u}\left(O_{1}\right) \cap \Pi_{1}^{-}$. It follows from (5) that in the neighbourhood $\Pi_{2}^{+}, s_{k 1}^{02} \equiv T_{12}\left(s_{k 1}^{11}\right)$ will accumulate to the segment $T_{12}\left(l_{u}^{1}\right) \cap \Pi_{2}^{+}$of the unstable manifolds of $O_{1}$. and hence will intersect the strips $\sigma_{i}^{02}$ at $i$, where $i \leq \bar{k}(\bar{k}$ not dependent on $\mu$ ). From (18) we obtain that the family $s_{k i 1}^{12} \equiv T_{02}^{i}\left(s_{k 1}^{02} \cap \sigma_{i}^{02}\right)$ on $\Pi_{2}^{-}$belongs to the strips $\sigma_{i}^{12}$ at all $i \leq \bar{k}$. By virtue of (18), the equation of $s_{k i 1}^{12}$ is $x_{12}=\gamma(\mu)^{-i} x_{2}^{+}(\mu)(1+\ldots)$.

It follows from (14) that for $\mu<\gamma(\mu)^{-i} x_{2}^{+}$the image $T_{21}\left(s_{k i 1}^{12}\right)$ has two transversal intersection with the stable manifold $W_{\text {loc }}^{s}\left(O_{1}\right)$. Hence, for sufficiently large $j, s_{1}(\mu)$ intersects the strip $\sigma_{j}^{01}$ along two 

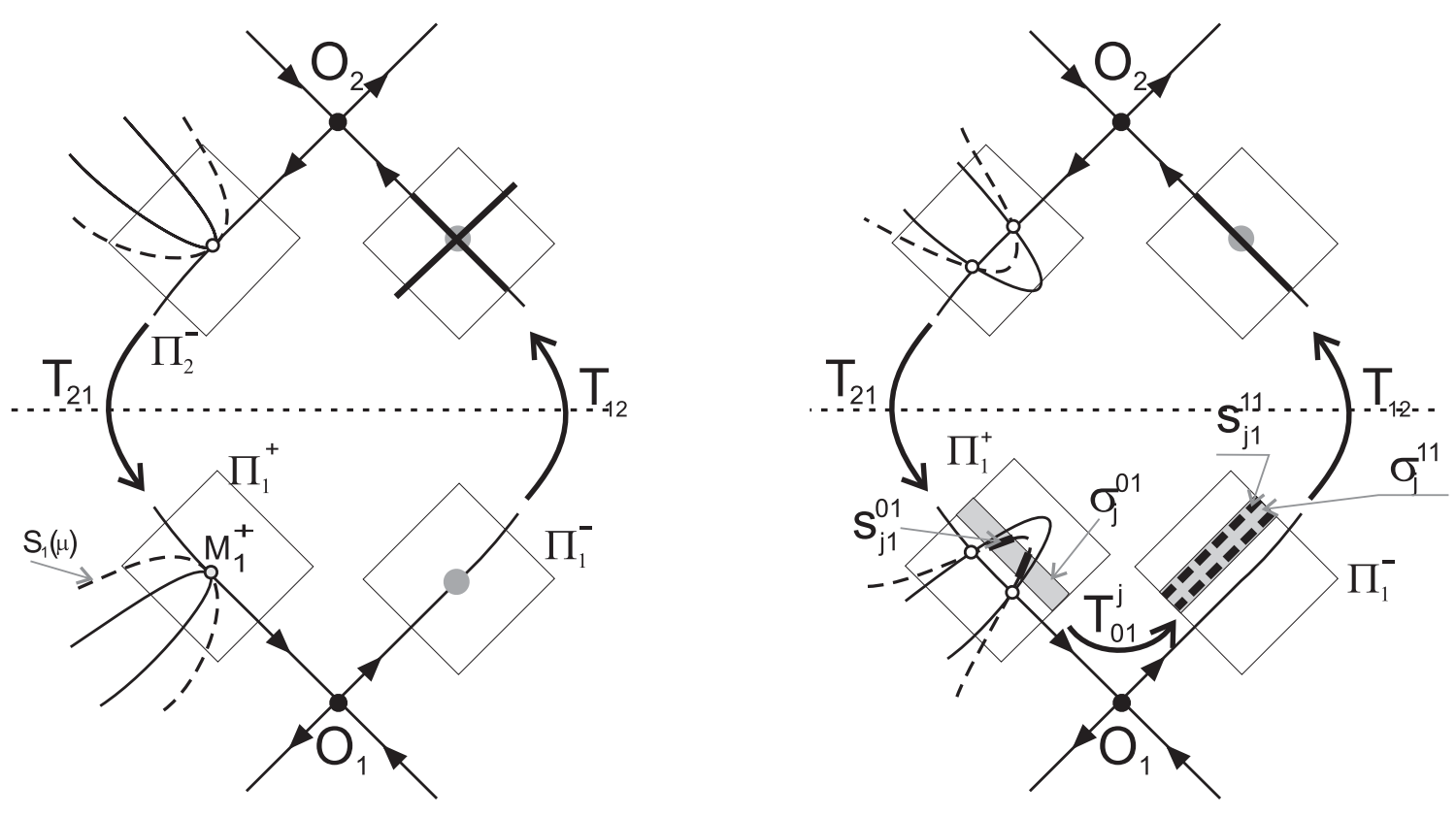

a) $\mu=0$

b) $\mu>0$

Figure 10:

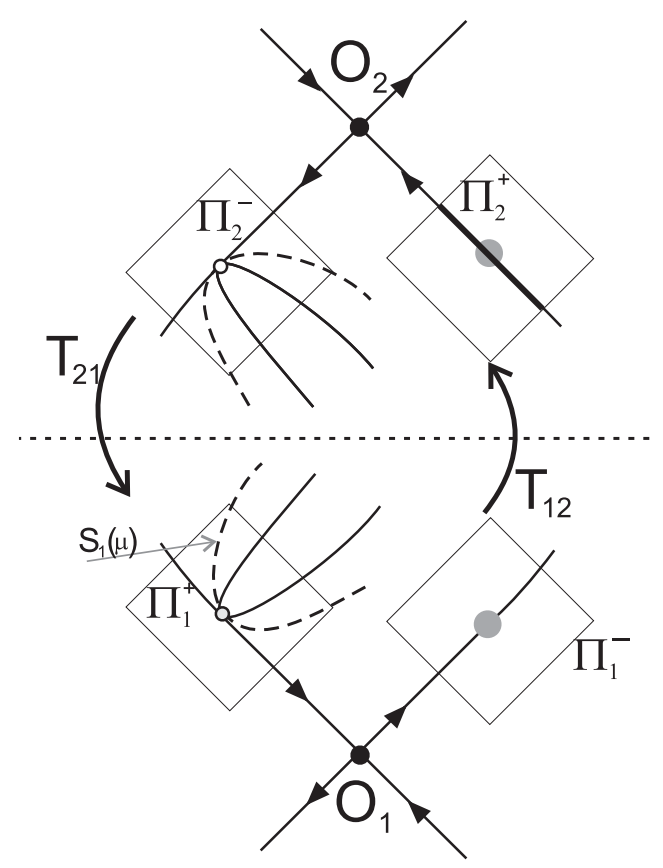

a) $\mu=0$

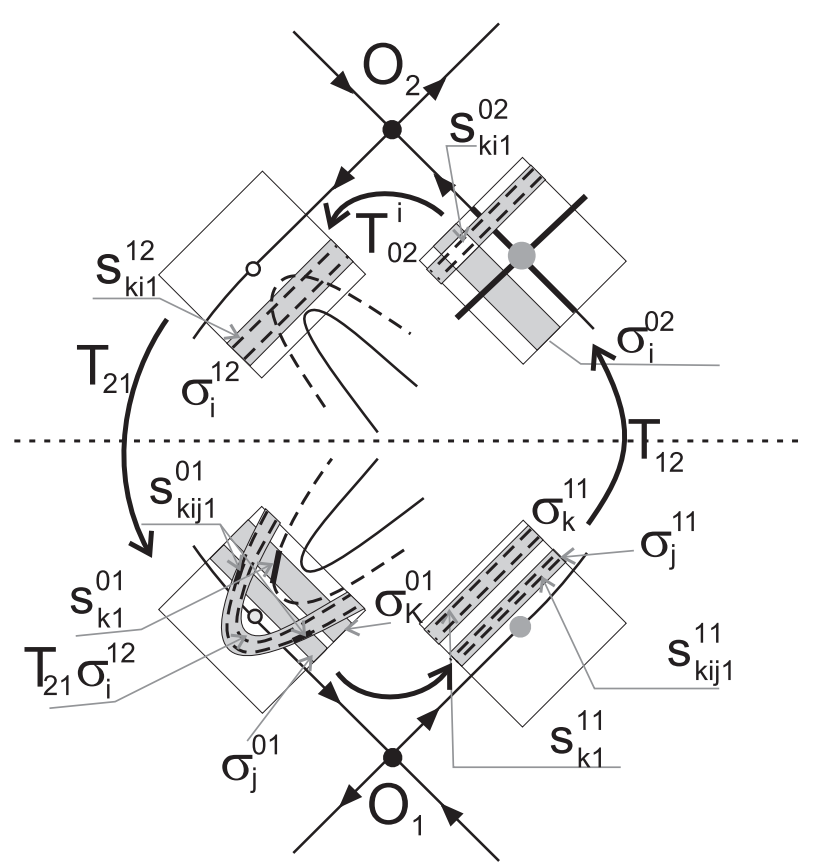

b) $\mu>0$

Figure 11: 
components $s_{k i j 1}^{01}$ and $s_{k i j 2}^{01}$, see Fig. 11b. By virtue of $(20)$, as $j \rightarrow \infty$, the curves $s_{k i j 1}^{11} \equiv T_{01}^{j}\left(s_{k i j 1}^{01}\right)$ on $\Pi_{1}^{-}$accumulate to the segment $l_{u}^{1} \equiv W_{\text {loc }}^{u}\left(O_{1}\right) \cap \Pi_{1}^{-}$. From (20) we can calculate the distance between $s_{j 1}^{11}$ and $W_{\text {loc }}^{u}\left(O_{1}\right)$ which is approximately equal to $x_{1}^{+} \lambda^{j}$. By construction, $s_{k i j 1}^{11}=f^{l}(\operatorname{Fix}(R)) \cap \Pi_{1}^{-}$, where $l=n_{2} / 2+k+n_{1}+i+n_{2}+j$.

Lemma 4 In the intervals $\delta_{i}$, the set of parameter values $\mu$ for which the family $f_{\mu}$ unfolds generically the symmetric type heteroclinic cycle, consisting of two saddle fixed points $\mathrm{O}_{1}$ and $\mathrm{O}_{2}$, heteroclinic orbits $\Gamma_{12}$ and new non-transversal heteroclinic orbits $\Gamma_{21}^{l 1}$, is dense.

Proof It follows from lemma 3 that in each of the intervals $\delta_{i}$ there is a dense set $\tilde{\delta}_{i}$ such that for all $\mu_{i}^{*} \in \tilde{\delta}_{i}$ the diffeomorphism $f_{\mu_{i}^{*}}$ has a homoclinic tangency with respect to fixed point $O_{1}$. According to Proposition 1 we can split this homoclinic tangency in such way that the diffeomorphism $f_{\mu_{i j}^{*}}$ will have a tangency of the unstable manifold of $O_{1}$ and the curve $f^{l}(\operatorname{Fix}(R)) \cap \Pi_{1}^{-}$, where $\mu_{i j}^{*}-\mu_{i}^{*}$ will be approximately equal to $x_{1}^{+} \lambda^{j}$, see the proof of Proposition 1. By reversibility, each such a tangency corresponds to the existence of a non-transversal heteroclinic cycle containing the points $O_{1}$ and $O_{2}$. Finally, by choosing $j$ sufficiently large, we prove the density of parameter values $\mu_{i j}^{*}$ in the interval $\delta_{i}(\mu)$.

Remark 3 It can be shown that Newhouse intervals $\delta_{i}$ in fact contain dense sets of diffeomorphisms having non-transversal symmetric heteroclinic cycles with tangencies of all possible types (see the classification of tangency with respect to the reciprocal position of the manifolds in Section 2.3).

In order to obtain such other types of tangency, we repeat the construction of proposition 1 but we choose the component $s_{j 2}^{01}$ instead $s_{j 1}^{01}$ in the case $d_{21}<0$ and the component $s_{k i j 2}^{01}$ instead $s_{k i j 1}^{01}$ in the case $d_{21}>0$.

\subsection{Density of diffeomorphisms with non-symmetric heteroclinic cycles}

Lemma 5 The set of parameter values $\mu^{*}$, such that $f_{\mu^{*}}$ has a nonsymmetric heteroclinic cycle $C=$ $\left\{O_{1}, O_{2}, \Gamma_{12}, \tilde{\Gamma}_{21}\right\}$, and $f_{\mu}$ unfolds the non-transversal heteroclinic orbits $\tilde{\Gamma}_{21}$ generically, is dense in $\delta_{i}$.

\section{Proof}

Let us choose the parameter $\mu^{*}$ such that the diffeomorphisms $f_{\mu^{*}}$ has the heteroclinic cycle which is depicted in Fig. 7b and Fig. 7d. In general, such diffeomorphisms are selected by the conditions $d_{21}>0$ and the sign of multipliers can be also negative. We claim that under small perturbation we can obtain a diffeomorphism with an "a priori non-symmetric heteroclinic" cycle (Fig. 8). For the diffeomorphisms with positive multipliers of saddle fixed points such heteroclinic cycle belongs to the third class. It is clear that in this case $f_{\mu^{*}}$ has a non-transversal heteroclinic point $M_{1 i j k}^{-}$which is close to $M_{1}^{-}$. Of course, the unstable manifold $W^{u}$ of the point $O_{2}$ intersects the strip $\sigma_{m}^{01}$ along two components $\omega_{m 1}^{01}$ and $\omega_{m 2}^{01}$ (Fig. 12) for sufficiently large $m$. Let us iterate $\omega_{m 1}^{01}$ :

$$
\begin{gathered}
\omega_{m 1}^{11}=T_{01}^{m}\left(\omega_{m 1}^{01}\right) ; \quad \omega_{m l 1}^{02}=T_{12}\left(\omega_{m 1}^{11}\right) \cap \sigma_{l}^{02} ; \\
\omega_{m l 1}^{12}=T_{02}^{l}\left(\omega_{m l 1}^{02}\right) ; \quad \omega_{m l 1}^{02}=T_{21}\left(\omega_{m l 1}^{12}\right) .
\end{gathered}
$$

The process of the iterations is illustrated in Fig. 12. It is easy to see that the curves $\omega_{m l 1}^{02}$ have the shape of a "parabola". By a small perturbation of $\mu$ (corresponding to a sufficiently large $l$, as $\mu-\mu^{*}=O\left(\gamma^{-l}\right)$ ) we can obtain a tangency of the "parabola" $\omega_{m l 1}^{02}$ (for a certain $l \geq \bar{k}$ ) and the segment of the stable manifold $W_{\text {loc }}^{s}\left(O_{1}\right) \cap \Pi_{1}^{+}$of $O_{1}$. It is readily verified that this tangency is associated with an "a priori non-symmetric heteroclinic cycle"

This completes the proof of parts $1-3$ of Theorem 1 . 


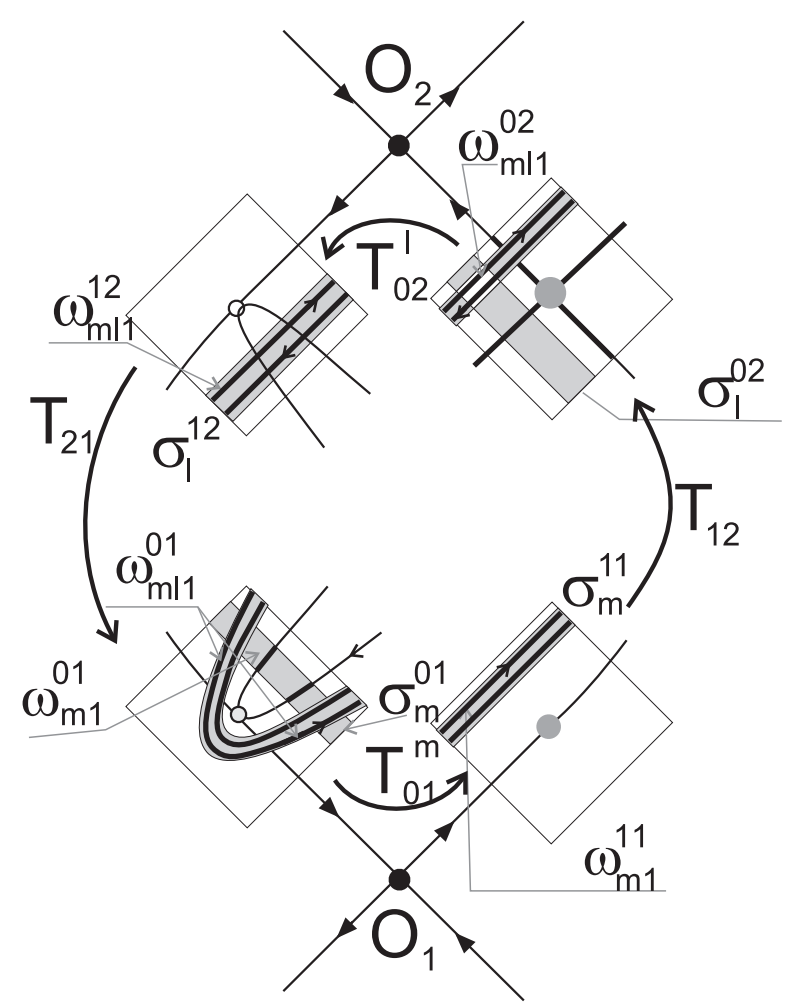

Figure 12:

\section{Bifurcations of single-round periodic orbits}

In this section we study bifurcations of single-round periodic orbits, lying entirely in $U$. Such orbits have exactly one intersection point with $\Pi_{l}^{+}$and $\Pi_{l}^{-}, l=1,2$. Let $\Lambda$ be some single-round periodic orbit and let $P_{l}^{+}$and $P_{l}^{-}$be its intersection points with $\Pi_{l}^{+}$and $\Pi_{l}^{-}$, respectively. Then, there exist some integers $i \geq \bar{k}$ and $j \geq \bar{k}$ such that

$$
P_{1}^{+} \in \sigma_{i}^{01}, P_{1}^{-} \in \sigma_{i}^{11}, P_{2}^{+} \in \sigma_{j}^{02}, P_{2}^{-} \in \sigma_{j}^{12} .
$$

Moreover, the following relations hold:

$$
P_{1}^{-}=T_{01}^{i}\left(P_{1}^{+}\right), P_{2}^{+}=T_{12}\left(P_{1}^{-}\right), P_{2}^{-}=T_{02}^{j}\left(P_{2}^{+}\right), P_{1}^{+}=T_{21}\left(P_{2}^{-}\right) .
$$

Thus, the point $P_{1}^{+}$can be considered as a fixed point of a first return map $T_{i j}(\mu)$ defined as the following composition of the local and global maps

$$
T_{i j} \equiv T_{21} \cdot T_{02}^{j} \cdot T_{12} \cdot T_{01}^{i}: \sigma_{i}^{01} \mapsto \Pi_{1}^{+} .
$$

The study of bifurcations of single-round periodic orbits in the family $f_{\mu}$ is reduced to the study of bifurcations of fixed points of the first return maps $T_{i j}(\mu)$ for various sufficiently large $i$ and $j$. Formulas (5),(14) and (18) make it possible to find an explicit expression for the map $T_{i j}(\mu)$. In fact, it turns out that by appropriate rescaling, $T_{i j}(\mu)$ can be put into a convenient standard form. This is the topic of the next sections.

\subsection{Construction of the Poincaré map $T_{i i}$ and Rescaling Lemma.}

In this section, we construct the first-return maps that describe symmetric periodic orbits. In order for a fixed point of $T_{i j}$ to be able to be a symmetric periodic point, it is easily verified that $i$ must be equal 
to $j$. Hence we first focus on $T_{i i}$. We have

$$
T_{i i}=T_{21} \circ T_{02}^{i} \circ T_{12} \circ T_{01}^{i}: \quad \sigma_{i}^{01} \rightarrow \Pi_{k}^{+} .
$$

It follows from (6), (15) and (19) that

$$
\begin{gathered}
T_{i i}^{-1}=T_{01}^{-i} \circ T_{12}^{-1} \circ T_{02}^{-i} \circ T_{21}^{-1}= \\
R_{12}^{-1} \circ T_{02}^{i} \circ R_{12} \circ R_{12}^{-1} \circ T_{12} \circ R_{12}^{-1} \circ R_{12} \circ T_{01}^{i} \circ R_{12}^{-1} \circ R_{12} \circ T_{21} R_{12}= \\
R_{12}^{-1} \circ T_{02}^{i} \circ T_{12} \circ T_{01}^{i} \circ T_{21} R_{12} .
\end{gathered}
$$

This means that there exists an

$$
R_{*}=T_{01}^{-i} \circ T_{12}^{-1} \circ T_{02}^{-i} \circ R_{12},
$$

such that

$$
T_{i i}^{-1}=R_{*} \circ T_{i i} \circ\left(R_{*}\right)^{-1} .
$$

Note that $R_{*}=\left(R^{*}\right)^{-1}$, and in general $R^{*}$ is nonlinear. But, special coordinates can be chosen such that in terms of these coordinates, $R^{*}$ is linear. In order to show this, we write the local and global maps, using a simplified representation of these maps, introducing functions $p, q, \Phi, F$ and $G$ that represent the right-hand sides of the first and second equations of (18), (12) and (14), respectively:

$$
\begin{aligned}
& T_{01}^{i}\left(\left(x_{01}, y_{01}\right) \rightarrow\left(x_{11}, y_{11}\right)\right):\left\{\begin{array}{l}
x_{11}=p\left(x_{01}, y_{11}\right), \\
y_{01}=q\left(x_{01}, y_{11}\right) ;
\end{array}\right. \\
& T_{12}\left(\left(\bar{x}_{11}, \bar{y}_{11}\right) \rightarrow\left(\bar{x}_{02}, \bar{y}_{02}\right)\right):\left\{\begin{array}{l}
\bar{x}_{02}-x_{2}^{+}=\Phi\left(x_{11}, \bar{y}_{02}\right), \\
y_{11}-x_{2}^{+}=\Phi\left(\bar{y}_{02}, x_{11}\right)
\end{array}\right. \\
& T_{02}^{i}\left(\left(\bar{x}_{02}, \bar{y}_{02}\right) \rightarrow\left(\bar{x}_{12}, \bar{y}_{12}\right)\right):\left\{\begin{array}{l}
\bar{y}_{02}=p\left(\bar{y}_{12}, \bar{x}_{02}\right), \\
\bar{x}_{12}=q\left(\bar{y}_{12}, \bar{x}_{02}\right) ;
\end{array}\right. \\
& T_{21}\left(\left(\bar{x}_{12}, \bar{y}_{12}\right) \rightarrow\left(\bar{x}_{01}, \bar{y}_{01}\right)\right):\left\{\begin{array}{l}
x_{01}-x_{1}^{+}=F\left(x_{12}, y_{12}-x_{1}^{+}, \mu\right), \\
y_{01}=G\left(x_{12}, y_{12}-x_{1}^{+}, \mu\right) .
\end{array}\right.
\end{aligned}
$$

The map $T_{01}^{i}$ is defined on the strip $\sigma_{i}^{01}=\Pi_{1}^{+} \cap T_{01}^{-i}\left(\Pi_{1}^{-}\right)$. Hence, without loss of generality we can choose the new coordinates $\left(\xi_{1}, \eta_{1}\right)$ on $\sigma_{i}^{01}$, where $\xi_{1}=x_{01}-x_{1}^{+}, \eta_{1}=y_{11}-x_{2}^{+}$. It is easy to see that subsequently

$$
\begin{aligned}
& \bar{x}_{01}=x_{1}^{+}+\xi_{1}, \\
& \bar{y}_{01}=q\left(x_{1}^{+}+\xi_{1}, x_{2}^{+}+\eta\right) .
\end{aligned}
$$

Analogously, on the strip $\sigma_{i}^{02}=\Pi_{2}^{+} \cap T_{02}^{-i}\left(\Pi_{2}^{-}\right)$we choose coordinates $\left(\bar{\xi}_{2}, \bar{\eta}_{2}\right)$, where $\bar{\xi}_{2}=x_{02}-x_{2}^{+}$, $\bar{\eta}_{2}=y_{12}-x_{1}^{+}$, so that

$$
\begin{aligned}
& \bar{x}_{02}=x_{2}^{+}+\bar{\xi}_{2}, \\
& \bar{y}_{02}=p\left(x_{1}^{+}+\bar{\eta}_{2}, x_{2}^{+}+\bar{\xi}_{2}\right) .
\end{aligned}
$$

Rewriting the maps $T_{01}^{i}, T_{12}, T_{02}^{i}$ and $T_{21}$ in terms of these coordinates, we obtain

$$
\begin{aligned}
& T_{01}^{i}\left(\left(\xi_{1}, \eta_{1}\right) \rightarrow\left(x_{11}, y_{11}\right)\right):\left\{\begin{array}{l}
x_{11}=p\left(x_{1}^{+}+\xi_{1}, x_{2}^{+}+\eta\right), \\
y_{11}=x_{2}^{+}+\eta ;
\end{array}\right. \\
& T_{12}\left(\left(\bar{x}_{11}, \bar{y}_{11}\right) \rightarrow\left(\bar{\xi}_{2}, \bar{\eta}_{2}\right)\right):\left\{\begin{array}{l}
\bar{\xi}_{2}=\Phi\left(x_{11}, p\left(x_{1}^{+}+\bar{\eta}_{2}, x_{2}^{+}+\bar{\xi}_{2}\right)\right), \\
y_{11}-x_{2}^{+}=\Phi\left(p\left(x_{1}^{+}+\bar{\eta}_{2}, x_{2}^{+}+\bar{\xi}_{2}\right), x_{11}\right)
\end{array}\right. \\
& T_{02}^{i}\left(\left(\bar{x}_{02}, \bar{y}_{02}\right) \rightarrow\left(\bar{x}_{12}, \bar{y}_{12}\right)\right):\left\{\begin{array}{l}
\bar{x}_{12}=q\left(x_{1}^{+}+\bar{\eta}_{2}, x_{2}^{+}+\bar{\xi}_{2}\right), \\
\bar{y}_{12}=x_{1}^{+}+\bar{\eta}_{2} ;
\end{array}\right. \\
& T_{21}\left(\left(\bar{x}_{12}, \bar{y}_{12}\right) \rightarrow\left(\overline{\bar{\xi}}_{1}, \overline{\bar{\eta}}_{1}\right)\right):\left\{\begin{array}{l}
\overline{\bar{\xi}}_{1}=F\left(\bar{x}_{12}, \bar{y}_{12}-x_{1}^{+}, \mu\right), \\
q\left(x_{1}^{+}+\overline{\bar{\xi}}_{1}, x_{2}^{+}+\overline{\bar{\eta}}_{1}\right)=G\left(\bar{x}_{12}, \bar{y}_{12}-x_{1}^{+}, \mu\right) .
\end{array}\right.
\end{aligned}
$$


Therefore, the map $T_{12} T_{01}^{i}:\left(\xi_{1}, \eta_{1}\right) \rightarrow\left(\bar{\xi}_{2}, \bar{\eta}_{2}\right)$ can be written in the form

$$
\begin{aligned}
& \bar{\xi}_{2}=\Phi\left(p\left(x_{1}^{+}+\xi_{1}, x_{2}^{+}+\eta_{1}\right), p\left(x_{1}^{+}+\bar{\eta}_{2}, x_{2}^{+}+\bar{\xi}_{2}\right)\right), \\
& \eta_{1}=\Phi\left(p\left(x_{1}^{+}+\bar{\eta}_{2}, x_{2}^{+}+\bar{\xi}_{2}\right), p\left(x_{1}^{+}+\xi_{1}, x_{2}^{+}+\eta_{1}\right)\right)
\end{aligned}
$$

It is easy to see that the last equations define $\bar{\xi}_{2}$ and $\eta_{1}$ uniquely as a function of $\xi_{1}$ and $\bar{\eta}_{2}$. Indeed, we can resolve this system with respect to $\left(\xi_{1}, \bar{\eta}_{2}\right)$, obtaining

$$
\begin{aligned}
& \bar{\xi}_{2}=\Psi\left(\xi_{1}, \bar{\eta}_{2}\right), \\
& \eta_{1}=\Psi\left(\bar{\eta}_{2}, \xi_{1}\right) .
\end{aligned}
$$

It follows from (26) that the map $T_{21} T_{02}^{i}:\left(\bar{\xi}_{2}, \bar{\eta}_{2}\right) \rightarrow\left(\overline{\bar{\xi}}_{1}, \overline{\bar{\eta}}_{1}\right)$ has the following form

$$
\begin{aligned}
& \overline{\bar{\xi}}_{1}=F\left(q\left(x_{1}^{+}+\bar{\eta}_{2}, x_{2}^{+}+\bar{\xi}_{2}\right), \bar{\eta}_{2}, \mu\right), \\
& q\left(x_{1}^{+}+\overline{\bar{\xi}}_{1}, x_{2}^{+}+\overline{\bar{\eta}}_{1}\right)=G\left(q\left(x_{1}^{+}+\bar{\eta}_{2}, x_{2}^{+}+\bar{\xi}_{2}\right), \bar{\eta}_{2}, \mu\right) .
\end{aligned}
$$

Now, we choose the new coordinates

$$
\xi=\xi_{1}, \quad \eta=\bar{\eta}_{2}
$$

on the strip $\sigma_{i}^{01}$, so that the maps $T_{12} T_{01}^{i}:(\xi, \eta) \rightarrow\left(\bar{\xi}_{1}, \bar{\eta}_{2}\right)$ and $T_{21} T_{02}^{i}:\left(\bar{\xi}_{2}, \bar{\eta}_{2}\right) \rightarrow(\overline{\bar{\xi}}, \overline{\bar{\eta}})$ can be rewritten as

$$
\begin{aligned}
& \bar{\xi}_{2}=\Psi(\xi, \eta), \\
& \bar{\eta}_{2}=\eta
\end{aligned}
$$

and

$$
\begin{aligned}
& \overline{\bar{\xi}}=F\left(q\left(x_{1}^{+}+\bar{\eta}_{2}, x_{2}^{+}+\bar{\xi}_{2}\right), \bar{\eta}_{2}, \mu\right), \\
& q\left(x_{1}^{+}+\overline{\bar{\xi}}, x_{2}^{+}+\Psi(\overline{\bar{\eta}}, \overline{\bar{\xi}})\right)=G\left(q\left(x_{1}^{+}+\bar{\eta}_{2}, x_{2}^{+}+\bar{\xi}_{2}\right), \bar{\eta}_{2}, \mu\right),
\end{aligned}
$$

respectively.

Let us substitute the values of $\bar{\xi}_{2}$ and $\bar{\eta}_{2}$ given by (30) into (31). Then we find that the map $T_{i i}$ has the form

$$
\begin{aligned}
& \overline{\bar{\xi}}=F\left(q\left(x_{1}^{+}+\eta, x_{2}^{+}+\Psi(\xi, \eta)\right), \eta, \mu\right), \\
& q\left(x_{1}^{+}+\overline{\bar{\xi}}, x_{2}^{+}+\Psi(\overline{\bar{\eta}}, \overline{\bar{\xi}})\right)=G\left(q\left(x_{1}^{+}+\eta, x_{2}^{+}+\Psi(\xi, \eta)\right), \eta, \mu\right) .
\end{aligned}
$$

It follows from the reversibility properties of $T_{21}$ (see $(6),(7),(8)$ ), that the functions $F$ and $G$ satisfy:

$$
\text { if }\left\{\begin{array} { l } 
{ z _ { 1 } ^ { * } = F ( z _ { 1 } , z _ { 2 } ) } \\
{ z _ { 2 } ^ { * } = G ( z _ { 1 } , z _ { 2 } ) }
\end{array} \text { then } \left\{\begin{array}{l}
z_{2}=F\left(z_{2}^{*}, z_{1}^{*}\right) \\
z_{1}=G\left(z_{2}^{*}, z_{1}^{*}\right)
\end{array}\right.\right.
$$

Hence, from (32) we obtain

$$
\begin{aligned}
& \eta=F\left(q\left(x_{1}^{+}+\overline{\bar{\xi}}, x_{2}^{+}+\Psi(\overline{\bar{\eta}}, \overline{\bar{\xi}})\right), \overline{\bar{\xi}}, \mu\right) \\
& q\left(x_{1}^{+}+\eta, x_{2}^{+}+\Psi(\xi, \eta)\right)=G\left(q\left(x_{1}^{+}+\overline{\bar{\xi}}, x_{2}^{+}+\Psi(\overline{\bar{\eta}}, \overline{\bar{\xi}})\right), \overline{\bar{\xi}}, \mu\right) .
\end{aligned}
$$

The equations (32) and (33) provide an implicit form of the maps $T_{i i}$ and $T_{i i}^{-1}$, respectively. From these expressions, one readily verifies the following result:

Proposition 2 In coordinates $(\xi, \eta)$ the first-return map $T_{i i}$ is reversible with respect to the linear involution $\tilde{R}$, where $\tilde{R}(\xi, \eta)=(\eta, \xi)$. That is,

$$
T_{i i}=\tilde{R} \circ T_{i i}^{-1} \circ \tilde{R}
$$


After normalizing the reversing symmetry, we now seek to normalize the map $T_{i i}$ further by a (rescaling) coordinate transformation. In order to preserve the $\tilde{R}$-reversibility, we require such a coordinate transformation to be $\tilde{R}$-equivariant. With $\tilde{R}$ as defined above, this implies that we allow only coordinate transformations of the form $(\xi, \eta) \rightarrow(X, Y)$, where $(X, Y)=(h(\xi, \eta), h(\eta, \xi))$. We obtain the following:

Lemma 6 (Rescaling Lemma) In some rescaled coordinates $(X, Y)$ the map $T_{i i}(\mu)$ is $\tilde{R}$-reversible and can be written in the following form

$$
\begin{aligned}
& \bar{X}=Y+\varepsilon_{1 i}, \\
& \bar{Y}=M-X-Y^{2}+\varepsilon_{2 i}
\end{aligned}
$$

where

$$
M=-d_{21} / A^{2}\left[\mu-2 x_{2}^{+} \gamma^{-i}+\ldots\right] \gamma^{2 i} \lambda^{-2 i},
$$

and $\varepsilon_{l i}, l=1,2$, denote some functions such that

$$
\left\|\varepsilon_{l i}(X, Y, M)\right\|_{C^{r-2}}=O\left(\gamma^{-i}\right) .
$$

In the rescaled coordinates $(X, Y)$, the domain where the map $T_{i i}$ is defined includes the disc $\|X, Y\| \leq K_{i i}$ where $K_{i i} \rightarrow+\infty$ as $i \rightarrow+\infty$.

Proof By Proposition 2, we know that the map $T_{i i}$ can be written in coordinates $(\xi, \eta)$, so that $T_{i i}$ is $\tilde{R}$-reversible. We first examine more closely this procedure, after which we carry out the rescaling procedures.

As discussed above, we choose the coordinates $\left(\xi_{1}, \eta_{1}\right)$ on $\sigma_{i}^{01}$, where $\xi_{1}=x_{01}-x_{1}^{+}, \eta_{1}=y_{11}-x_{2}^{+}$ and the coordinates $\left(\bar{\xi}_{2}, \bar{\eta}_{2}\right)$ on $\sigma_{i}^{02}$, where $\bar{\xi}_{2}=x_{02}-x_{2}^{+}, \bar{\eta}_{2}=y_{12}-x_{1}^{+}$.

The map $T_{21} T_{02}^{i}:\left(\bar{\xi}_{2}, \bar{\eta}_{2}\right) \rightarrow\left(\overline{\bar{\xi}}_{1}, \overline{\bar{\eta}}_{1}\right)$ is implicitly given by (27). It follows from (13), (18), and (26), that

$$
p(u, v)=\lambda(\mu)^{i} u\left(1+\gamma^{-i} p_{i}(u, v, \mu)\right)
$$

and

$$
\Phi(u, v, \mu)=A(\mu) u+B(\mu) v+O\left(u^{2}+|u v|+v^{2}\right) .
$$

Substituting these expressions for the functions $p$ and $\Phi$ into (27), we obtain

$$
\begin{aligned}
\bar{\xi}_{2}= & A \lambda^{i} x_{1}^{+}(1+\ldots)+B \lambda^{i} x_{1}^{+}(1+\ldots)+A \lambda^{i} \xi_{1}(1+\ldots)+ \\
& B \lambda^{i} \bar{\eta}_{2}(1+\ldots)+O\left(\left|\lambda^{i} \gamma^{-i}\right|\left(\left|\eta_{1}\right|+\left|\bar{\xi}_{2}\right|+\xi_{1}^{2}+\bar{\eta}_{2}^{2}+\left|\xi_{1} \bar{\eta}_{2}\right|\right)\right) \\
\eta_{1}= & A \lambda^{i} x_{1}^{+}(1+\ldots)+B \lambda^{i} x_{1}^{+}(1+\ldots)+A \lambda^{i} \bar{\eta}_{2}(1+\ldots)+ \\
& B \lambda^{i} \xi_{1}(1+\ldots)+O\left(\left|\lambda^{i} \gamma^{-i}\right|\left(\left|\bar{\xi}_{2}\right|+\left|\eta_{1}\right|+\bar{\eta}_{2}^{2}+\xi_{1}^{2}+\left|\bar{\eta}_{2} \xi_{1}\right|\right)\right)
\end{aligned}
$$

where the dots stand for the constant terms (i.e. independent of $\xi, \eta$ ) which tend to zero as $i \rightarrow \infty$, and have asymptotical order $\gamma^{-i}$.

It is easy to see that it is possible to resolve $\bar{\xi}_{2}$ and $\eta_{1}$ from (36), giving

$$
\begin{aligned}
\bar{\xi}_{2}= & A \lambda^{i} x_{1}^{+}(1+\ldots)+B \lambda^{i} x_{1}^{+}(1+\ldots)+A \lambda^{i} \xi_{1}(1+\ldots)+ \\
& B \lambda^{i} \bar{\eta}_{2}(1+\ldots)+O\left(\left|\lambda^{i} \gamma^{-i}\right|\left(\xi_{1}^{2}+\bar{\eta}_{2}^{2}+\left|\xi_{1} \bar{\eta}_{2}\right|\right)\right) \\
\eta_{1}= & A \lambda^{i} x_{1}^{+}(1+\ldots)+B \lambda^{i} x_{1}^{+}(1+\ldots)+A \lambda^{i} \bar{\eta}_{2}(1+\ldots)+ \\
& B \lambda^{i} \xi_{1}(1+\ldots)+O\left(\left|\lambda^{i} \gamma^{-i}\right|\left(\bar{\eta}_{2}^{2}+\xi_{1}^{2}+\left|\bar{\eta}_{2} \xi_{1}\right|\right)\right)
\end{aligned}
$$


Now we consider the map $T_{21} T_{02}^{i}:\left(\bar{\xi}_{2}, \bar{\eta}_{2}\right) \rightarrow\left(\overline{\bar{\xi}}_{1}, \overline{\bar{\eta}}_{1}\right)$. Schematically, it is represented by the system of equations (29). From (14), (18), and (26), we have expressions for the functions $F, G$ and $q$ :

$$
\begin{gathered}
F(u, v)=-\frac{a_{21}}{2} \mu(1+O(\mu))+a_{21}(\mu) u+\left(1+b_{\mu} \mu\right) v+O\left(u^{2}+|u v|+v^{2}\right), \\
G(u, v)=\mu-\left(1-c_{\mu} \mu\right) u+d_{21}(\mu) v^{2}+O\left(u^{2}+|u v|+v^{3}\right)
\end{gathered}
$$

and

$$
q(u, v)=\gamma(\mu)^{-i} v\left(1+\gamma^{-i} q_{i}(u, v, \mu)\right) .
$$

Substituting these expressions into (29), we obtain that $T_{21} T_{02}^{i}$ takes the form

$$
\begin{gathered}
\overline{\bar{\xi}}_{1}=-\frac{a_{21}}{2} \mu(1+O(\mu))+a_{21} \gamma^{-i} x_{2}^{+}(1+\ldots)+a_{21} \gamma^{-i} \bar{\xi}_{2}(1+\ldots) \\
+\left(1+b_{\mu} \mu\right) \bar{\eta}_{2}+O\left(\gamma^{-2 i} \bar{\xi}_{2}^{2}+\gamma^{-2 i}\left|\bar{\xi}_{2} \bar{\eta}_{2}\right|+\bar{\eta}_{2}^{2}\right), \\
\gamma^{-i} x_{2}^{+}(1+\ldots)+\gamma^{-i} \overline{\bar{\eta}}_{1}(1+\ldots)\left[1+O\left(\left|\gamma^{-i}\right|\left(\left|\overline{\bar{\eta}}_{1}\right|+\left|\overline{\bar{\xi}}_{1}\right|\right)\right)\right]+O\left(\gamma^{-2 i} \overline{\bar{\xi}}_{1}\right) \\
=\mu-\left(1-c_{\mu} \mu\right) \gamma^{-i} x_{2}^{+}(1+\ldots)-\left(1-c_{\mu} \mu\right) \gamma^{-i} \bar{\xi}_{1}(1+\ldots)+ \\
O\left(\gamma^{-2 i} \bar{\eta}_{2}\right)+d_{21} \bar{\eta}_{2}^{2}(1+\ldots)+O\left(\gamma^{-2 i} \bar{\xi}_{2}^{2}+\gamma^{-2 i}\left|\bar{\xi}_{2} \bar{\eta}_{2}\right|+\left|\bar{\eta}_{2}\right|^{3}\right) .
\end{gathered}
$$

Like before, we choose the new coordinates

$$
\xi=\xi_{1}, \quad \eta=\bar{\eta}_{2}
$$

on the strip $\sigma_{i}^{01}$ and construct the composition of $T_{12} T_{01}^{i}$ and $T_{21} T_{02}^{i}$. We obtain

$$
\begin{gathered}
\overline{\bar{\xi}}=-\frac{a_{21}}{2} \mu(1+O(\mu))+a_{21} \gamma^{-i} x_{2}^{+}(1+\ldots)+a_{21} \gamma^{-i} \lambda^{i} \xi(1+\ldots) \\
+\left(1+b_{\mu} \mu+\ldots\right) \eta+O\left(|\lambda|^{i} \gamma^{-2 i} \xi+\left|\lambda^{i} \gamma^{-2 i} \xi \eta\right|+\eta^{2}\right) \\
\gamma^{-i} x_{2}^{+}(1+\ldots)+\gamma^{-i} \lambda^{i} A \overline{\bar{\eta}}(1+\ldots)\left[1+O\left(\left|\gamma^{-i}\right|(|\overline{\bar{\eta}}|+|\overline{\bar{\xi}}|)\right)\right]+\underline{O\left(\gamma^{-2 i} \overline{\bar{\xi}}\right)} \\
=\mu-\left(1-c_{\mu} \mu\right) \gamma^{-i} x_{2}^{+}(1+\ldots)-\left(1-c_{\mu} \mu\right) \gamma^{-i} \lambda^{i} \xi(1+\ldots)+ \\
\underline{O\left(\gamma^{-2 i} \eta\right)}+d_{21} \eta^{2}(1+\ldots)+O\left(\gamma^{-2 i} \xi^{2}+\gamma^{-2 i}|\bar{\xi} \eta|+|\eta|^{3}\right) .
\end{gathered}
$$

Subsequently, we eliminate the underlined terms in the second equation of (39), using the following construction: we substitute the expression for $\overline{\bar{\xi}}$ which is given by the first equation of (39) into the lefthand side of the second equation of (39). If we differentiate both sides of the obtained second equation of (39) with respect to $\eta$, then - since $d_{21} \neq 0$ - the resulting equation defines $\eta$ uniquely as a smooth function of $\xi$ and $\overline{\bar{\eta}}$ :

$$
\eta=\Theta(\xi, \overline{\bar{\eta}})=O\left(\gamma^{-2 i}\right) .
$$

By construction, the following shift of the origin of the coordinates

$$
\xi^{\text {new }}=\xi-\xi^{*}, \quad \eta^{\text {new }}=\eta-\eta^{*},
$$


where $\eta^{*}=\Theta\left(\xi^{*}, \eta^{*}\right)$, eliminates the underlined terms in (39). In order to preserve reversibility we have to choose the shift such that $\xi^{*}=\eta^{*}$. It turns out that we do so. Indeed, let us consider the system of equations

$$
\Theta(\xi, \eta)=\eta, \xi=\eta
$$

It is easy to see that it has a unique solution $\left(\xi=\eta^{*}, \eta=\eta^{*}\right)$ where $\eta^{*}=O\left(\gamma^{-2 i}\right)$.

By the shift

$$
\xi^{\text {new }}=\xi-\eta^{*}, \quad \eta^{\text {new }}=\eta-\eta^{*},
$$

we bring the system (39) into the form

$$
\begin{gathered}
\overline{\bar{\xi}}=-\frac{a_{21}}{2} \mu(1+O(\mu))+a_{21} \gamma^{-i} x_{2}^{+}(1+\ldots)+a_{21} \gamma^{-i} \lambda^{i} \xi(1+\ldots) \\
+\left(1+b_{\mu} \mu+\ldots\right) \eta+O\left(|\lambda|^{i} \gamma^{-2 i} \xi+\left|\lambda^{i} \gamma^{-2 i} \xi \eta\right|+\eta^{2}\right) \\
\gamma^{-i} \lambda^{i} A \overline{\bar{\eta}}(1+\ldots)\left(1+O\left(\left|\gamma^{-i}\right|(|\overline{\bar{\eta}}|+|\overline{\bar{\xi}}|)\right)\right)= \\
{\left[\mu-(2+O(\mu)+\ldots) \gamma^{-i} x_{2}^{+}\right]-\left(1-c_{\mu} \mu\right) \gamma^{-i} \lambda^{i} \xi(1+\ldots)+} \\
d_{21} \eta^{2}(1+\ldots)+O\left(\gamma^{-2 i} \xi^{2}+\left|\gamma^{-2 i} \bar{\xi} \eta\right|+|\eta|^{3}\right)
\end{gathered}
$$

The obtained map is also reversible because such type of coordinate shift does not destroy reversibility.

Note that all the constant (zero order) terms in the second equation of (40) are collected in the square brackets. For $\mu \approx 2 \gamma^{-i} x_{2}^{+}(1+\ldots)$ it can be sufficiently small. Suppose that $M_{i}=\left[\mu-(2+O(\mu)+\ldots) \gamma^{-i} x_{2}^{+}\right]=$ $O\left(\lambda^{2 i} \gamma^{-2 i}\right)$. Consider the zero order terms $M_{1 i}$ in the first equation of (40). Since $\mu=(2+\ldots) \gamma^{-i} x_{2}^{+}$, then $M_{1 i}=-\frac{a_{21}}{2} \mu(1+O(\mu))+a_{21} \gamma^{-i} x_{2}^{+}(1+\ldots)$ has indefinite order of smallness. In order to estimate $M_{1 i}$ we use the property of reversibility. It is easy to see that $T_{i i}$ transfer the point $(\xi=0, \eta=0)$ to $\left(\xi^{*}, \eta^{*}\right)$, where $\xi^{*}=M_{1 i}, \eta^{*}=\frac{M_{i}}{A \gamma^{-i} \lambda^{i}}(1+\ldots)=O\left(\gamma^{-i} \lambda^{i}\right)$. According to reversibility, the point $\left(\eta^{*}, \xi^{*}\right)$ should be transferred to the point $(0,0)$. Due to $(40)$, we obtain that

$$
0=M_{1 i}+a_{21} \frac{M_{i}}{A}(1+\ldots)+M_{1 i}+\ldots
$$

Hence, $M_{1 i}=-a_{21} \frac{M_{i}}{2 A}(1+\ldots)$. We obtain that $M_{1 i}$ has the same order of smallness.

By a further rescaling of variables

$$
\begin{aligned}
& \xi=-A / d_{21} \lambda^{i} \gamma^{-i} X \\
& \eta=-A / d_{21} \lambda^{i} \gamma^{-i} Y
\end{aligned}
$$

we bring system (40) to the form

$$
\begin{aligned}
& \bar{X}=Y+O\left(\gamma^{-i}\right) \\
& \bar{Y}\left(1+O\left(\gamma^{-i}\right)\right)=M-X-Y^{2}+O\left(\gamma^{-i}\right)
\end{aligned}
$$

where

$$
M=-\left(\mu-2 \gamma^{-i} x_{2}^{+}+\ldots\right) d_{21} / A^{2} \lambda^{-2 i} \gamma^{2 i} .
$$

Now we can resolve the last system with respect to variables $\bar{X}$ and $\bar{Y}$. We obtain (34). The lemma is proved. 


\subsection{Bifurcations of the reversible Henon map}

The first-return map $T_{i i}$ is close to the reversible Henon map of the form

$$
\begin{gathered}
\bar{X}=Y \\
\bar{Y}=M-X-Y^{2}
\end{gathered}
$$

whose bifurcations have been well studied, so that it is possible to recover the bifurcation picture for the initial map $T_{i i}$. Indeed, bifurcations of single-round periodic orbits in the family $f_{\mu}$, or -equivalently bifurcations of fixed points of maps $T_{i i}$ with sufficiently large $i$, can be studied if to know the structure of bifurcations of fixed points of map (43). It is easy to verify that the map (43) has a fixed point with double multipliers +1 at $M=-1$ and double multipliers -1 at $M=3$. This map has no fixed points if $M<-1$. Moreover, we have an elliptic fixed point at $M \in(-1,3)$. At the parameter value $M=M(\psi)=\cos ^{2} \psi-2 \cos \psi$ the fixed point has multipliers $\nu_{1,2}=e^{ \pm i \psi}$. The fixed point is a generic (Liapunov stable) elliptic point if $\psi \notin\{\pi / 2, \arccos (-1 / 4), \pi / 3\}$ (cf [1]). The map has an generic elliptic fixed point $O_{i}$ at $-1+\ldots<M<3+\ldots$, where $M \notin\{-3 / 4,0,9 / 16\}$. By the rescaling lemma, similar properties hold for the map $T_{i i}$. Using (42), it then follows that

Proposition 3 In the parameter space there exist infinite many intervals $\delta(\mu)_{i}^{e l}$ with boundary points

$$
\begin{aligned}
& \mu_{i}^{+1}=2 \gamma^{-i} x_{2}^{+}(1+\ldots)+\frac{A^{2}}{d_{21}} \gamma^{-2 i} \lambda^{2 i} \\
& \mu_{i}^{-1}=2 \gamma^{-i} x_{2}^{+}(1+\ldots)-\frac{3 A^{2}}{d_{21}} \gamma^{-2 i} \lambda^{2 i}
\end{aligned}
$$

accumulating to $\mu=0$. For values of parameter $\mu$ from these intervals, the diffeomorphism $f_{\mu}$ has elliptic periodic points.

\subsection{Bifurcations of non-symmetric periodic orbits. Rescaling lemma for the map $T_{i j}$}

Now we consider non-symmetric single-round periodic orbits. Such orbits stay much more time near one saddle point than near the other. Recall that the study of bifurcations of single-round periodic orbits in the family $f_{\mu}$ may be reduced to the study of bifurcations of fixed points of the first return maps $T_{i j}(\mu), T_{i j}(\mu)=T_{21} T_{02}^{j} T_{12} T_{01}^{i}$, for various sufficiently large $i$ and $j$. In order to study non-symmetric periodic orbits we should suppose that $i \neq j$. In particular, we will focus on the case $i>j$. From (12), (14), and (18), we can find the explicit form of the map $T_{i j}(\mu)$ in the initial coordinates. We can rescale these coordinates and parameters in order to put the map $T_{i j}(\mu)$ in some standard form. The following rescaling lemma holds.

Lemma 7 Let $i>j$. In some rescaled coordinates $(X, Y)$ the map $T_{i j}\left(\mu_{1}, \mu_{2}\right)$ can be written in the following form

$$
\begin{aligned}
& \bar{X}=Y, \\
& \bar{Y}=M-Y^{2}+O\left((\lambda \gamma)^{(i-j)}\right),
\end{aligned}
$$

where

$$
M=-\frac{d_{21}}{A^{2}}\left[\mu-x_{2}^{+} \gamma^{-i}+\ldots\right] \gamma^{2 i} \lambda^{-2 j},
$$

Here, the dots stand for terms which tend to zero as $i, j \rightarrow \infty$ (and which are independent of $(X, Y)$ ). In the rescaled coordinates $(X, Y)$, the domain where the map $T_{i j}$ is defined includes the disc $\|X, Y\| \leq K_{i j}$ where $K_{i j} \rightarrow+\infty$ as $i, j \rightarrow+\infty$.

This lemma is a particular case of the rescalling lemma in $[12]^{2}$. For selfcontainment we provide a proof, simplifying the exposition of [12].

\footnotetext{
${ }^{2}$ Note that in this lemma we do not require to preserve reversibility.
} 
Proof Like we did before, we will use $\left(\xi_{1}, \eta_{1}\right)$ and $\left(\xi_{2}, \eta_{2}\right)$ as the coordinates on the strips $\sigma_{i}^{01}$ and $\sigma_{j}^{02}$ respectively, where

$$
\begin{array}{ll}
x_{01}-x_{1}^{+}=\xi_{1} & x_{02}-x_{2}^{+}=\xi_{2}, \\
y_{11}-x_{2}^{+}=\eta_{1} & y_{12}-x_{1}^{+}=\eta_{2} .
\end{array}
$$

Then, by virtue of (18) and (12), the map $T_{12} T_{01}^{i}:\left(\xi_{1}, \eta_{1}\right) \mapsto\left(\bar{\xi}_{2}, \bar{\eta}_{2}\right)$ is written as

$$
\begin{gathered}
\bar{\xi}_{2}=A \lambda^{i} x_{1}^{+}(1+\ldots)+A \lambda^{i}(1+\ldots) \xi_{1}+B \lambda^{j} x_{1}^{+}(1+\ldots) \bar{\eta}_{2}+ \\
O\left(\lambda^{i} \gamma^{-i}\left(\left|\eta_{1}\right|+\left|\bar{\xi}_{2}\right|+\xi_{1}^{2}+\bar{\eta}^{2}+\left|\xi_{1} \bar{\eta}_{2}\right|\right),\right. \\
\eta_{1}=A \lambda^{j} x_{1}^{+}(1+\ldots)+A \lambda^{j}(1+\ldots) \bar{\eta}_{2}+B \lambda^{i} x_{1}^{+}(1+\ldots) \\
+B \lambda^{i}(1+\ldots) \xi_{1}+O\left(\lambda^{i} \gamma^{-i}\left(\left|\eta_{1}\right|+\left|\bar{\xi}_{2}\right|+\xi_{1}^{2}+\bar{\eta}^{2}+\left|\xi_{1} \bar{\eta}_{2}\right|\right) ;\right.
\end{gathered}
$$

(Note that when deriving (48) we took into account that $\lambda^{2 i} \leq \lambda^{i} \gamma^{-i}, \lambda_{2}^{2 j} \leq \gamma^{-j} \lambda^{j}$.) According to Implicit Function Theorem we ca resolve these equations with respect to variables $\bar{\xi}_{2}$ and eta $_{1}$. We obtain

$$
\begin{array}{r}
\bar{\xi}_{2}=A \lambda^{i} x_{1}^{+}(1+\ldots)+A \lambda^{i}(1+\ldots) \xi_{1}+\lambda^{j} x_{1}^{+}(1+\ldots) \bar{\eta}_{2}+ \\
\psi_{1}^{i j}\left(\xi_{1}, \eta_{1}\right), \\
\eta_{1}=A \lambda^{j} x_{1}^{+}(1+\ldots)+A \lambda^{j}(1+\ldots) \bar{\eta}_{2}+B \lambda^{i} x_{1}^{+}(1+\ldots) \\
+B \lambda^{i}(1+\ldots) \xi_{1}+\psi_{2}^{i j}\left(\xi_{1}, \eta_{1}\right) ;
\end{array}
$$

where

$$
\psi_{l}^{i j}\left(\xi_{1}, \eta_{1}\right),=O\left(\lambda^{i} \gamma^{-i}\left(\xi_{1}^{2}+\bar{\eta}^{2}+\left|\xi_{1} \bar{\eta}_{2}\right|\right)\right), \quad l=1,2 .
$$

It follows from (14) and (18) that the map $T_{21} T_{02}^{j}:\left(\bar{\xi}_{2}, \bar{\eta}_{2}\right) \mapsto\left(\overline{\bar{\xi}}_{1}, \overline{\bar{\eta}}_{1}\right)$ is written as

$$
\begin{gathered}
\overline{\bar{\xi}}_{1}=-\frac{a_{21}}{2} \mu(1+O(\mu))+a_{21} \gamma^{-j} x_{2}^{+}(1+\ldots)+a_{21} \gamma^{-j}(1+\ldots) \bar{\xi}_{2}+ \\
(1+\ldots) \bar{\eta}_{2}+\psi_{3}^{i j}\left(\bar{\xi}_{2}, \bar{\eta}_{2}\right), \\
\gamma^{-i} x_{2}^{+}(1+\ldots)+\gamma^{-i} \overline{\bar{\eta}}_{1}\left(1+\gamma^{-i} O\left(\overline{\bar{\xi}}_{1}+\overline{\bar{\eta}}_{1}\right)\right)+\gamma^{-2 i} O\left(\overline{\bar{\xi}}_{1}\right)=\mu- \\
\gamma^{-j} x_{2}^{+}(1+\ldots)-\gamma^{-j}(1+\ldots) \bar{\xi}_{2}+d_{21}(1+\ldots) \bar{\eta}_{2}^{2}+\psi_{4}^{i j}\left(\bar{\xi}_{2}, \bar{\eta}_{2}\right) ;
\end{gathered}
$$

where the dots stand for the constant (i.e. independent on $\xi$ and $\eta$ ) terms which tend to zero as $i, j \rightarrow \infty$. By $\psi_{3}^{i j}$ and $\psi_{4}^{i j}$ we denote the functions of the following orders:

$$
\begin{aligned}
& \psi_{3}^{i j}\left(\bar{\xi}_{2}, \bar{\eta}_{2}\right)=O\left(\left|\gamma^{-2 j}\right| \bar{\xi}_{2}^{2}+\left|\gamma^{-j} \bar{\xi}_{2} \bar{\eta}_{2}\right|+\left|\bar{\eta}_{2}^{2}\right|\right), \\
& \psi_{4}^{i j}\left(\bar{\xi}_{2}, \bar{\eta}_{2}\right)=O\left(\left|\gamma^{-2 j}\right| \bar{\xi}_{2}^{2}+\left|\gamma^{-j} \bar{\xi}_{2} \bar{\eta}_{2}\right|+\left|\bar{\eta}_{2}^{3}\right|\right) .
\end{aligned}
$$

Let us substitude the expression for $\bar{\xi}_{2}$ and $\eta_{1}$ which is given by the system (49) into right side of the first equation of (50) and left side of the second equation of (50) respectively. Then, in new coordinates $\xi^{\text {new }}=\xi_{1}$ and $\eta^{\text {new }}=\bar{\eta}_{2}$ the first-return map $T_{21} T_{02}^{j} T_{12} T_{01}^{i}:(\xi, \eta) \mapsto(\overline{\bar{\xi}}, \overline{\bar{\eta}})$ can be written in following 
form

$$
\begin{array}{r}
\overline{\bar{\xi}}=-\frac{a_{21}}{2} \mu(1+O(\mu))+a_{21} \gamma^{-j} x_{2}^{+}(1+\ldots)+a_{21} A \lambda^{i} \gamma^{-j}(1+\ldots) \xi+ \\
(1+\ldots) \eta+\psi_{5}^{i j}(\xi, \eta), \\
A \lambda^{j} \gamma^{-i} \overline{\bar{\eta}}\left(1+\gamma^{-i} O(\overline{\bar{\xi}}+\overline{\bar{\eta}})\right)+\gamma^{-2 i} O(\overline{\bar{\xi}})=\mu-\gamma^{-j} x_{2}^{+}(1+\ldots)+ \\
A \lambda^{i} \gamma^{-j}(1+\ldots) \xi+O\left(\gamma^{-2 i}\right) \eta+d_{21}(1+\ldots) \eta^{2}+\psi_{6}^{i j}(\xi, \eta) ;
\end{array}
$$

where

$$
\begin{aligned}
& \psi_{5}^{i j}(\xi, \eta)=O\left(\left|\lambda^{i} \gamma^{-i-j}\right| \xi^{2}+\left|\lambda^{i} \gamma^{-j} \xi \eta\right|+\left|\eta^{2}\right|\right), \\
& \psi_{6}^{i j}(\xi, \eta)=O\left(\left|\lambda^{i} \gamma^{-i-j}\right| \xi^{2}+\left|\lambda^{i} \gamma^{-j} \xi \eta\right|+\left|\eta^{3}\right|\right) .
\end{aligned}
$$

(we took into account that $\lambda^{i}<\gamma^{-i}, \lambda^{j}<\gamma^{-j}, i<j, \ldots$ )

Now we substitute into the left-hand side of the second equation of (51) the expression for $\overline{\bar{\xi}}$ which is given by the first equation of (51). If we differentiate both sides of the obtained second equation with respect to $\eta$, then the resulting equation, since $d_{21} \neq 0$, defines $\eta$ uniquely as a smooth function of $\xi$ and $\overline{\bar{\eta}}$ :

$$
\eta=\varphi(\xi, \overline{\bar{\eta}})=O\left(\left|\gamma^{-2 i}\right|+\left|\gamma^{-j}\right|\right)
$$

It is easy to see then, that if we write, schematically, the first equation of (51) in the form $\overline{\bar{\xi}}=\rho(\xi, \eta)$, then the system

$$
\eta=\varphi(\xi, \eta), \quad \xi=\rho(\xi, \eta)
$$

will have a unique solution $\left(\xi^{*}, \eta^{*}\right)$ where

$$
\xi^{*}, \eta^{*}=O\left(\left|\gamma^{-2 i}\right|+\left|\gamma^{-j}\right|+|\mu|\right) .
$$

By construction, the following shift of the origin of the coordinates:

$$
\xi^{\text {new }}=\xi-\xi^{*}, \quad \eta^{\text {new }}=\eta-\eta^{*}
$$

brings the system (51) to the form

$$
\begin{gathered}
\overline{\bar{\xi}}=a_{21} \gamma^{-j} x_{2}^{+}(1+\ldots)+a_{21} A \lambda^{i} \gamma^{-j}(1+\ldots) \xi+(1+\ldots) \eta+\psi_{5}^{i j}(\xi, \eta), \\
A \lambda^{j} \gamma^{-i} \overline{\bar{\eta}}\left(1+\gamma^{-i} O(\overline{\bar{\xi}}+\overline{\bar{\eta}})\right)=\left[\mu-\gamma^{-j} x_{2}^{+}+\ldots\right]+ \\
A \lambda^{i} \gamma^{-j}(1+\ldots) \xi+d_{21}(1+\ldots) \eta^{2}+\psi_{6}^{i j}(\xi, \eta)
\end{gathered}
$$

Note that we have now nullified the constant (zero order) terms in (52) and in the first equation and the linear (first order) in $\eta$ and $\overline{\bar{\xi}}$ term in the second equation of (52) is also made zero now. All zero order terms in the second equation of (52) are collected in the square brackets.

Now, we rescale variables as follows

$$
\xi=\alpha X \quad \eta=\beta Y
$$

where

$$
\beta=-\gamma^{-i} \lambda^{j} \frac{A}{d_{21}}(1+\ldots) \quad \alpha=-\gamma^{-i} \lambda^{j} \frac{A}{d_{21}}(1+\ldots)
$$


We obtain the following system:

$$
\begin{aligned}
& \bar{X}=Y+O\left(\lambda^{i} \gamma^{-j}\right) \\
& \bar{Y}\left(1+O\left(\lambda^{i} \gamma^{-j}\right)\right)=M(1+\ldots)-Y^{2}+O\left((\lambda \gamma)^{i-j}\right) .
\end{aligned}
$$

where

$$
M=-\left[\mu-\gamma^{-i} x_{2}^{+}+\ldots\right] \frac{d_{21}}{A^{2}} \gamma^{2 i} \lambda^{-2 j} .
$$

It is easy to verify that after small (order $O\left(\lambda^{i} \gamma^{-j}\right)$ ) nonlinear transformations of variables $U$ and $V$ we can bring the system (53) to the form

$$
\begin{aligned}
& \bar{X}=Y \\
& \bar{Y}=M^{\text {new }}-Y^{2}+O\left((\lambda \gamma)^{i-j}\right),
\end{aligned}
$$

where

$$
M^{\text {new }}=-\left[\mu-\gamma^{-i} x_{2}^{+}+\ldots\right] \frac{d_{21}}{A^{2}} \gamma^{2 i} \lambda^{-2 j}
$$

The lemma is proven.

The map (45) is close to the one-dimensional parabola map

$$
\bar{y}=M-y^{2}
$$

whose bifurcations have been well studied. For the parabola map, the bifurcation set is contained in the interval $\left[-\frac{1}{4}, 2\right]$ of values of $M:$ at $M=-\frac{1}{4}$ there exists a fixed point with multiplier equal to +1 . This fixed point is attractive when $M \in\left(-\frac{1}{4}, \frac{3}{4}\right)$ and it undergoes a period-doubling bifurcation at $M=\frac{3}{4}$.

Combining these results, with Lemma 7 , we obtain that $T_{i j}$ has similar bifurcations as the parabola map (55). The map has an attractive fixed point $O_{i j}$ at $\mu$ from intervals $\delta(\mu)_{i}^{s u}$ with boundary points

$$
\begin{aligned}
& \mu_{i j}^{+1}=x_{2}^{+} \gamma^{-i}(1+\ldots)+\frac{A^{2}}{4 d_{21}} \lambda^{2 j} \gamma^{-2 i} \\
& \mu_{i j}^{-1}=x_{2}^{+} \gamma^{-i}(1+\ldots)-\frac{3 A^{2}}{4 d_{21}} \lambda^{2 j} \gamma^{-2 i}
\end{aligned}
$$

which arises at the saddle-node bifurcation at $\mu=\mu_{k}^{+1}$ and loses stability at $\left.\mu=\mu_{k}^{-1}\right)$ through a perioddoubling bifurcation. We thus obtain

Proposition 4 In the parameter space there exist infinitely many intervals $\delta(\mu)_{i}^{\text {su }}$ accumulating to $\mu=0$, such that for values of parameter $\mu$ from these intervals, the diffeomorphism $f_{\mu}$ has stable and unstable periodic points.

\subsection{Diffeomorphisms with infinitely many stable, unstable and elliptic periodic or- bits in Newhouse regions}

We now can combine the above results into the following proposition, providing also the proof of the fourth part of Theorem 1 .

Proposition 5 On $\delta_{j}^{1}$ the values of $\mu$, for which $f_{\mu}$ simultaneously has infinite many stable, and infinite many completely unstable and infinite many elliptic periodic orbits are dense.

Proof Consider arbitrarily $\mu_{j}$ from interval $\delta_{j}$. Then, it follows from lemma 4 that there exists, arbitrarily close to $\mu_{j}$, a new $\mu=\mu_{j 1}$ such that $f_{\mu_{j 1}}$ has a non-transversal heteroclinic cycle $C_{j}=$ $\left\{O_{1}, O_{2}, \Gamma_{12}, \Gamma_{21 j}\right\}$. It follows from proposition 3 that in any neighbourhood of the point $\mu_{j 1}$ there exists a an interval $\delta_{1,0}^{(j)} \in \delta_{j}$ of values of $\mu$ such that for $\mu \in \delta_{1,0}^{(j)}$ the family $f_{\mu}$ has an elliptic periodic orbit. 
Furthermore, on the interval $\delta_{1,0}^{(j)}$, by virtue of proposition 4 and density of diffeomorphisms with nontransversal heteroclinic cycle, there exists an interval $\delta_{1,1}^{(j)} \subset \delta_{1,0}^{(j)}$ such that for $\mu \in \delta_{1,1}^{(j)}$ the family $f_{\mu}$ has elliptic and stable - unstable periodic orbits, which lie in $U$. Similarly, on the interval $\delta_{1,1}^{(j)}$ we find a subinterval $\delta_{2,2}^{(j)}$ such that for $\mu \in \delta_{2,2}^{(j)}$ the diffeomorphism $f_{\mu}$ has two elliptic orbits, two completely unstable and two stable periodic orbits lying in $U$.

Finally we obtain a countable set of nested intervals

$$
\delta_{1,1}^{(j)} \supset \delta_{2,2}^{(j)} \supset \ldots \supset \delta_{n, n}^{(j)} \supset \ldots
$$

such that for $\mu \in \delta_{n, n}^{(j)}$ the diffeomorphism $f_{\mu}$ simultaneously has $n$ elliptic, $n$ stable and $n$ completely unstable periodic orbits. This completes the proof of Proposition5, and in turn of Theorem 1.

\section{Acknowledgements}

It is a great pleasure to thank S.V. Gonchenko, L.P. Shilnikov and D.V. Turaev for helpful discussions. This research has been supported by the UK EPSRC, through an Advanced Research Fellowship (JSWL), the UK Royal Society, through a postdoctoral fellowship (OVS), and INTAS (grant No. 2000-221), RFBR (No. 02-01-00273), CRDF (No 13314) (OVS). We would like to thank IMPA (Rio de Janeiro) for their hospitality during a visit, in which part of this paper was written. During this visit, JSWL was supported by a UK Royal Society exchange grant.

\section{References}

[1] Biragov V.S. Bifurcations in a two-parameter family of conservative mappings that are close to the Henon mapping. (Russian) Translated in Selecta Math. Soviet. 9, 1990, no. 3, pp. 273-282. Methods of the qualitative theory of differential equations (Russian), 10-24, Gor'kov. Gos. Univ., Gorki, 1987 11)

[2] Champneys A.R.. Homoclinic orbits in reversible systems and their applications in mechanics, fluids and optics. Physica D 112 (1998), 158-186.

[3] Duarte P. Abundance of elliptic isles at conservative bifurcations. Dyn. Stability Systems, 1999, vol. 14, pp. 339-356

[4] Duarte P. Persistent homoclinic tangencies for conservative maps near the identity. Ergod.Th. \&6 Dynam.Sys., 2000, vol.20, No.2, pp. 393-438.

[5] Gavrilov, N.K., and Shilnikov, L.P., On three-dimensional dynamical systems close to systems with a structurally unstable homoclinic curve. Part 1, Math.USSR Sb., 1972, vol.17, pp.467-485 ; Part 2, Math.USSR Sb, 1973, vol.19, pp.139-156.

[6] Gonchenko, V.S., About existence of attractors and repellers in reversible systems with additional symmetry. Proceedings of conference, NNGU, Nyzhny Novgorod, in Russian, 2003, pp.98-100.

[7] Gonchenko, S.V., Moduli of $\Omega$-conjugacy of two-dimensional diffeomorphisms with a structurally unstable heteroclinic contour. Sbornik: Mathematics, 1996, vol.187:9, pp.1261-1281.

[8] Gonchenko, S.V., and Shilnikov, L.P., Invariants of $\Omega$-conjugacy of diffeomorphisms with a structurally unstable homoclinic trajectory. Ukrainian Math.J., 1990, vol.42, No.2, pp.134-140.

[9] Gonchenko, S.V., and Shilnikov, L.P., On the moduli of systems with a non-rough Poincaré homoclinic curve. Russian Acad. Sci. Izv. Math., 1993, vol.41, No.3, pp.417-445. 
[10] Gonchenko, S.V., and Shilnikov, L.P., On two-dimensional analytical area-preserving diffeomorphisms with infinitely many stable elliptic periodic points. Regular and Chaotic Dynamics , 1997, v.2, No. 3, pp. 106-123 (in Russian). Gonchenko,

[11] Gonchenko, S.V., and Shilnikov, L.P., On two-dimensional area-preserving diffeomorphisms with infinitely many elliptic islands. J.Stat.Phys., 2000, v.101, No.1/2, pp.321-356.

[12] Gonchenko, S.V., Shilnikov, L.P., and Stenkin, O.V. On Newhouse regions with infinite many stable and unstable invariant tori. In: Progress in Nonlinear Science. Proc. of the Int. Conf. dedicated to A.A. Andronov, Vol. I: Mathematical problems of nonlinear dynamics. University of Nizhny Novgorod, Russia. pp 80-102 (2002).

[13] Gonchenko, S.V., Shilnikov, L.P., and Turaev, D.V., On Models with non-Rough Poincaré Homoclinic Curves. Physica D, 1993, vol. 62, N 1-4, pp. 1-14.

[14] Gonchenko S.V., Shilnikov L.P., Turaev D.V. Elliptic periodic orbits near a homoclinic tangency in four-dimensional symplectic maps and Hamiltonian systems with three degrees of freedom.- Regular and Chaotic Dynamics, 1998, vol. 3, No.4, pp. 3-26.

[15] Gonchenko S.V., Shilnikov L.P., Turaev D.V. Infinitely many elliptic periodic orbits in fourdimensional symplectic maps with a homoclinic tangency. WIAS-Preprint No.791, Berlin 2002. (to be publised in Proc. Steklov Inst. Math.)

[16] Gonchenko, S.V., Turaev, D.V., and Shilnikov, L.P. On models with a structurally unstable homoclinic curve. Soviet Math.Dokl., 1992, vol.44, No.2, 422-426.

[17] Gonchenko, S.V., Turaev, D.V., and Shilnikov, L.P. On the existence of Newhouse regions near systems with a structurally unstable homoclinic Poincaré curve (multidimensional case), Russian Acad. Sci.Dokl.Math., 1993, vol. 47, No.2, pp. 268-273.

[18] Gonchenko, S.V., Turaev, D.V., and Shilnikov, L.P. Dynamical phenomena in multi-dimensional systems with a structurally unstable homoclinic Poincaré curve. Russian Acad. Sci. Dokl. Math., 1993, vol. 47, No.3, pp.410-415.

[19] Gonchenko, S.V., Turaev, D.V., and Shilnikov, L.P. Homoclinic tangencies of arbitrary high orders in the Newhouse regions. J.Math.Sci., 2001, vol. 105, pp. 1738-1778.

[20] Gonchenko, S.V., Turaev, D.V., and Shilnikov, L.P. On Newhouse domains of two-dimensional diffeomorphisms which are close to a diffeomorphism with a structurally unstable heteroclinic cycle. Proc. of the Steklov Inst. of Math., 1997, vol.216, pp.7-118.

[21] Iooss, G., Travelling water-waves, as a paradigm for bifurcations in reversible infinite-dimensional "dynamical" systems. Proceedings of the International Congress of Mathematicians, Vol. III (Berlin, 1998). Doc. Math. (1998), Extra Vol. III, 611-622.

[22] Lamb, J.S.W., and Roberts, J.A.G., Time-reversal symmetry in dynamical systems: a survey. Physica D, 1998, vol. 112, pp. 1-39.

[23] Lamb, J.S.W., Teixeira, M.A., and Webster, K.N., Heteroclinic cycle bifurcations near Hopf-zero bifurcation in 3D reversible vector fields. Preprint DynamIC 2003-10.

[24] Michelson, D., Steady solutions of the Kuramoto-Sivashinsky equation. Physica D, 1986, vol. 19, pp. $89-111$.

[25] Newhouse. S.E. Diffeomorphisms with infinitely many sinks. Topology, 1974, vol. 13, pp.9-18. 
[26] Newhouse, S.E., The abundance of wild hyperbolic sets and non-smooth stable sets for diffeomorphisms. Publ. Math. IHES., 1979, vol. 50, pp. 101-151.

[27] Newhouse, S.E., Nondensity of Axiom A(a) on $S^{2}$. Proc. A.M.S. Symp. Pure Math., 1970, vol. 14, pp. 191-202.

[28] Palis, J., and Viana, M. High dimension diffeomorphisms displaying infinitely many sinks. Ann. Math., 1994, vol. 140, pp. 207-250.

[29] Topaj D. and Pikovsky A. Reversibility vs. synchronization in oscillator lattices. Physica D, 2002, vol. 170, pp. 118-130.

[30] Romero N., Persistence of homoclinic tangencies in higher dimensions. Ergod. Th. \& Dynam.Sys., 1995, vol. 15, pp. 735-757. 